\title{
Visual-Motor Transformations Required for Accurate and Kinematically Correct Saccades
}

\author{
J. DOUGLAS CRAWFORD ${ }^{1}$ AND DANIEL GUITTON ${ }^{2}$ \\ ${ }^{1}$ Centre for Vision Research and Departments of Psychology and Biology, York University, Toronto, Ontario M3J 1P3; \\ and ${ }^{2}$ Montreal Neurological Institute and Department of Neurology and Neurosurgery, McGill University, Montreal, \\ Quebec H3A 2B4, Canada
}

Crawford, J. Douglas and Daniel Guitton. Visual-motor transformations required for accurate and kinematically correct saccades. J. Neurophysiol. 78: 1447-1467, 1997. The goal of this study was to identify and model the three-dimensional (3-D) geometric transformations required for accurate saccades to distant visual targets from arbitrary initial eye positions. In abstract 2-D models, target displacement in space, retinal error (RE), and saccade vectors are trivially interchangeable. However, in real 3-D space, RE is a nontrivial function of objective target displacement and 3-D eye position. To determine the physiological implications of this, a visuomotor "lookup table" was modeled by mapping the horizontal/vertical components of RE onto the corresponding vector components of eye displacement in Listing's plane. This provided the motor error (ME) command for a 3-D displacement-feedback loop. The output of this loop controlled an oculomotor plant that mechanically implemented the position-dependent saccade axis tilts required for Listing's law. This model correctly maintained Listing's law but was unable to correct torsional position deviations from Listing's plane. Moreover, the model also generated systematic errors in saccade direction (as a function of eye position components orthogonal to $\mathrm{RE}$ ), predicting errors in final gaze direction of up to $25^{\circ}$ in the oculomotor range. Plant modifications could not solve these problems, because the intrisic oculomotor input-output geometry forced a fixed visuomotor mapping to choose between either accuracy or Listing's law. This was reflected internally by a sensorimotor divergence between input-defined visual displacement signals (inherently 2-D and defined in reference to the eye) and output-defined motor displacement signals (inherently 3-D and defined in reference to the head). These problems were solved by rotating RE by estimated 3-D eye position (i.e., a reference frame transformation), inputting the result into a 2D-to-3-D "Listing's law operator,' and then finally subtracting initial 3-D eye position to yield the correct ME. This model was accurate and upheld Listing's law from all initial positions. Moreover, it suggested specific experiments to invasively distinguish visual and motor displacement codes, predicting a systematic position dependence in the directional tuning of RE versus a fixed-vector tuning in ME. We conclude that visual and motor displacement spaces are geometrically distinct such that a fixed visual-motor mapping will produce systematic and measurable behavioral errors. To avoid these errors, the brain would need to implement both a 3-D position-dependent reference frame transformation and nontrivial 2-D-to-3-D transformation. Furthermore, our simulations provide new experimental paradigms to invasively identify the physiological progression of these spatial transformations by reexamining the position-dependent geometry of displacement code directions in the superior colliculus, cerebellum, and various cortical visuomotor areas.

\section{INTRODUCTION}

To generate accurate movements, the brain must transform visual information about the external world into motor commands for the internal world of body muscles (Andersen et al. 1983; Flanders et al. 1992). For some behaviors, i.e., rapid eye movements (saccades) to visual targets, several key steps in this process have been identified. For example, the visual input for saccades is retinal error (RE, stimulation of some point on the retina relative to the fovea), which is coded topographically in visual areas like V1 and the superficial layers of the superior colliculus (Sparks 1988; Wurtz and Albano 1980). At a subsequent level of processing, the deep layers of the superior colliculus possess a topographically similar map of saccade displacement commands, i.e., motor error (ME) (e.g., Freedman et al. 1996; Munoz et al. 1991; Robinson 1972; Schiller and Stryker 1972). Finally, reticular formation burst neurons (Henn et al. 1989; Luschei and Fuchs 1972) provide a velocity-like signal that activates motoneurons during saccades and is "neurally integrated" to generate the tonic signal that holds final position (Robinson 1975). However, the computational transformations between these well-defined levels, i.e., the sensorimotor transformations, remain the subject of considerable controversy.

Much of this controversy has centered around two classic models of the saccade generator. According to the spatial model (Fig. 1A) RE is added onto an internal representation of current eye position (orientation) to generate a desired eye position command, which is then compared with current eye position during the saccade to derive the instantaneous ME command that drives burst neurons until the eye is on target (Zee et al. 1976). In contrast, the displacement model (Fig. $1 B$ ) maps RE directly onto an initial ME command (Jürgens et al. 1981) without requiring internal comparisons with, or construction of, eye position signals. The distinction between these two models is important for two reasons. First, the models suggest two different mechanisms for mapping visuomotor space: reconstruction of target direction relative to the head (Fig. 1A) versus a succession of displacement signals defined relative to current eye position (Fig. $1 B$ ). Second, the displacement model posits a fixed sensorimotor mapping, i.e., a stimulus-response "lookup table," whereas the spatial model posits a (potentially) more flexible position-dependent transformation.

Unfortunately, one-dimensional (1-D) and 2-D versions of these models predict very similar behavior and have 


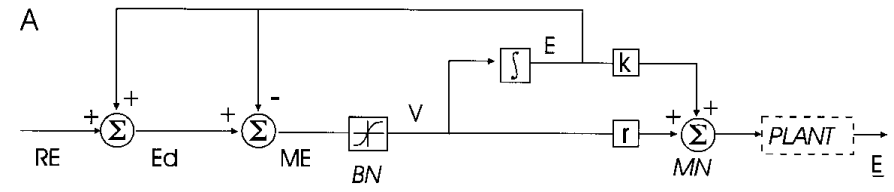

B

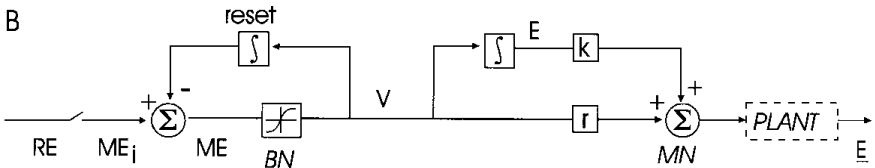

FIG. 1. One-dimensional (1-D) models of saccade generator. $A$ : spatial model (e.g., Zee et al. 1976). Retinal error (RE) is added to feedback copy of internal representation of eye position $(E)$ to obtain desired eye position $\left(E_{\mathrm{d}}\right)$. During saccade, another feedback copy of $E$ is subtracted from $E_{\mathrm{d}}$ to determine instantaneous motor error (ME). ME is multiplied by gain factor (up to physiologically determined saturation) by reticular formation burst neurons $(B N)$ whose output is eye velocity $(\mathrm{V})$. This signal is multiplied by internal estimate of plant viscosity constant (r) in its direct path to motoneurons $(M N)$ and is converted into $E$ by mathematical integration in indirect path. After multiplication by internal estimate of plant elasticity constant $(\mathrm{k}), E$ is also input to motoneurons, which drive plant to obtain actual eye position $(E)$. $B$ : displacement-feedback model. Difference in this model is that once decision is made to look at a certain target, RE maps trivially onto initial $\mathrm{ME}\left(\mathrm{ME}_{\mathrm{i}}\right)$, because they are geometrically indistinguishable in 1-D. Burst neuron output is now converted into current displacement history by integrator whose content is reset to 0 after each saccade. Subtracting this from $\mathrm{ME}_{\mathrm{i}}$ gives $\mathrm{ME}$ that guides and terminates saccade (Jürgens et al. 1981)

proven difficult to distinguish neurophysiologically. This is illustrated by the following arguments for the spatial and displacement hypotheses. First (displacement hypothesis), although some direct connections between the sensory and motor maps of the colliculus do exist (Moschovakis and Highstein 1994), the majority are indirect and complex (spatial hypothesis), including much of the visuomotor cortex (Sparks 1988; Wurtz and Albano 1980). Second (displacement hypothesis), other than in a few exceptional cases, saccade-related activity in the cortex overwhelmingly encodes displacements (reviewed in Moschovakis and Highstein 1994). Nevertheless (spatial hypothesis), many of these codes (notably those in posterior parietal cortex) possess eye-position-dependent "gain fields" (Andersen et al. 1985) that theoretically could produce the transformations necessary for the spatial hypothesis (Zipser and Andersen 1988). However (displacement hypothesis), some investigators are not convinced by this argument, even citing the relative subtlety of these gain fields as evidence against the spatial hypothesis (Moschovakis and Highstein 1994). Third (spatial hypothesis), the original displacement model (Fig. $1 B$ ) did not account for our ability to saccade toward remembered visual targets after an intervening saccade (Hallet and Lightstone 1976; Schlag et al. 1989; Sparks and Mays 1983 ). However (displacement hypothesis), more recent 2-D displacement models simulate such behavior by subtracting a vector representation of the intervening saccade from the original RE vector (Goldberg and Bruce 1990; Moschovakis and Highstein 1994; Waitzman et al. 1991), perhaps by shifting target representation within retinotopic cortical maps (Duhamel et al. 1992).

One limitation of the above studies is that they they have strictly employed abstract 2-D representations of real 3-D space. Some investigators have suggested that the displacement model is not consistent with the constraints observed in 3-D eye positions and rotational axes, i.e., Listing's law (Nakayama 1975; Sparks et al. 1987; Westheimer 1973). In particular, it has been suggested that the correct axes of rotation for Listing's law can only be computed through internal comparisons of current and desired 3-D eye position (Crawford and Vilis 1991). For example, Tweed and Vilis (1987, 1990b) modeled the colliculus ME command as a fixed-axis rotation computed by an upstream comparison between current and desired 3-D eye position on Listing's plane. However, this model was contradicted by subsequent stimulation studies of the superior colliculus, leading to the conclusion that Listing's law is implemented downstream from the colliculus (Hepp et al. 1993; Van Opstal et al. 1991). Subsequent investigations have thus focused on the late neuromuscular stages of 3-D saccade generation (Schnabolk and Raphan 1994; Straumann et al. 1995; Tweed et al. 1994 ), and opinions remain polarized between the view that Listing's law is a trivial, perhaps muscular phenomenon (Demer et al. 1995; Schnabolk and Raphan 1994) and the view that Listing's law poses an important problem for neural control (Crawford and Vilis 1995; Hepp 1994; Tweed et al. 1994).

The goal of the current investigation was to formulate the best possible 3-D versions of both the displacement and spatial models by identifying and incorporating all of the nontrivial geometric transformations required to take retinal stimulation (from distant targets) to a 3-D saccade. Moreover, these models were required to be consistent with the currently available physiological data (e.g., Crawford and Vilis 1992; Van Opstal et al. 1991). In the process of developing these models, we rigorously evaluated several conflicting ideas about the physiological implementation of Listing's law (Demer et al. 1995; Schnabolk and Raphan 1994; Tweed and Vilis 1990b; Van Opstal et al. 1991), but our main focus was the implications of this 3-D geometry for saccade accuracy, a subject that has received surprisingly little attention. The key theme that arose in this investigation was that the geometric properties of the eye and its movements dictate that RE and ME differ along two geometric criteria, making a simple stimulus-response lookup table problematic for both saccade accuracy and kinematics and implicating specific alternative solutions. To reach these conclusions and their important implications for visuomotor neurophysiology, we begin with an intuitive geometric analysis of retinal stimulation, eye position, and saccade axes in 3-D.

\section{B A C K GR O U N D}

\section{Describing $R E$ in $3-D$}

An important (but standard) assumption behind this study is that saccades are so fast that visual feedback is essentially absent during the movement. Thus, when we speak of RE, we refer strictly to visual information available before movement initiation. Moreover, for simplicity we will only consider distant visual targets and a cyclopean eye. Figure 2 illustrates RE as a 2-D oculocentric quantity specified by stimulation of some unique site on the retina. The location 


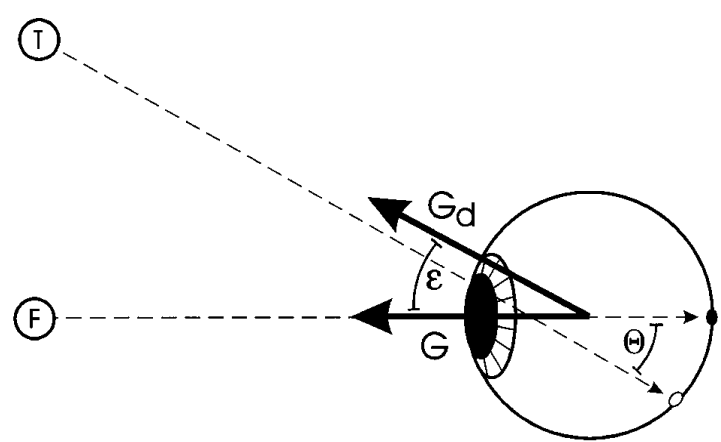

FIG. 2. Definition of RE. Eye is viewed from above. F, currently fixated target; T, potential target. - - - incident light rays that pass through optical node; $\bullet$, fovea, stimulated by light from $\mathrm{F}$; $\odot$, point on retina stimulated by light from $\mathrm{T}$, which is displaced to right of eye; $\Theta$, angle between incident light rays from $\mathrm{T}$ and $\mathrm{F} ; \mathrm{G}$, current gaze direction vector (heavy arrow ); $\mathrm{G}_{\mathrm{d}}$, desired gaze direction vector; $\epsilon$, angle between $\mathrm{G}$ and $\mathrm{G}_{\mathrm{d}}$.

of this site $(\bigcirc)$ relative to the fovea $(\bullet)$ is proportionate to the angle $\Theta$ between the incident light rays to these two sites, thus specifying both the magnitude and direction of the target displacement relative to current gaze direction $(\mathrm{G})$. Indeed, for all but the nearest targets, $\Theta$ is indistinguishable from $\epsilon$, the angle between $G$ and a second vector giving desired gaze direction $\left(G_{d}\right)$. It is for this reason that $\mathrm{RE}$ is useful in specifying desired gaze direction.

Because RE is initially represented in the brain as sites on a map (i.e., a lookup table), it can be interpreted in many ways by downstream structures. Each point on the retinal map specifies the horizontal and vertical projections of $\Theta$ (Fig. 2), $\Theta_{\mathrm{h}}$ and $\Theta_{\mathrm{v}}$. For the purposes of generating a displacement command, these values can be used to specify a rotation of gaze direction about the axis orthogonal to the plane containing current and desired gaze direction. However, for the purposes of computing target direction or desired eye position, it is preferable to note that the open circle specifies the vertical and horizontal components of desired gaze in eye coordinates (which will also look like a displacement from a head-fixed perspective). Other representations are possible, but they all share two important properties dictated by the geometric limitations of their input: RE is fundamentally 2-D (i.e., it does not specify the orientation of the eye about the desired gaze direction) and defined relative to the eye (the oculocentric reference frame).

\section{Listing's law and the degrees of freedom problem}

As illustrated in the preceding text, RE specifies desired gaze direction but not the angle of eye rotation about this "visual axis." Because the eye is capable of rotating about the visual axis from any initial position (e.g., Crawford and Vilis 1991; Henn et al. 1989), this poses a well-known computational problem for generating 3-D saccades: the degrees of freedom problem (Crawford and Vilis 1995). The oculomotor system utilizes Listing's law to determine this third, otherwise unspecified degree of freedom during saccades (Ferman et al. 1987; von Helmholtz 1925; Nakayama 1983; Tweed and Vilis 1990a). Listing's law states that if we take a static "snapshot" of eye position at any one time, it will be rotated from any arbitrarily chosen reference position about an axis that lies within a specific head-fixed plane. At one unique reference position (primary position), gaze is orthogonal to the associated plane of axes, which in this case is called Listing's plane (von Helmholtz 1925).

Listing's plane has been easier to visualize since the advent of the technology for recording 3-D eye position vectors (illustrated in Figs. 6-9 and 13). These vectors are parallel to the axis that would rotate the eye most directly from primary position to current position, and their length is proportionate to the magnitude of this rotation. Henceforth we will describe such vectors in a head-fixed, orthogonal coordinate system where the torsional axis is parallel to the primary gaze direction and clockwise/counterclockwise rotations are defined from the subject's perspective. With these conventions, Listing's law simply states that torsional eye position must equal zero. This predicted planar range of position vectors has now been visualized and confirmed numerous times in both humans and primates (e.g., Crawford and Vilis 1991; Tweed and Vilis 1990a; Van Opstal et al. 1991).

\section{Oculomotor reference frame problem}

Numerous investigators have also pointed out that the oculocentric geometry of RE poses a reference frame problem for visual perception and motor control, i.e., we often might want to know target position relative to the head (the craniotopic reference frame) rather than the eye. It has been suggested that this problem is solved by comparing raw visual signals with extraretinal eye position signals (e.g., Haustein and Mittelstaedt 1990; von Helmholtz 1925; Howard 1982) as in the 1-D spatial model (Zee al. 1976). However, the displacement-feedback model (Jürgens et al. 1981) and its variations (Moschovakis and Highstein 1994; Scudder 1988; Waitzman et al. 1991) seem to obviate this problem by mapping RE directly onto motor displacement commands. Why bother with positions and reference frames if motor systems only need to know which direction to move and how far (Woodwoth 1899)?

Figure 3 raises a possible problem for the latter view. Because fixed points on the retina are difficult to visualize, we have instead illustrated visual targets that are fixed with respect to the retina and thus stimulate fixed points on the retina. Various visual targets are viewed from head-fixed perspectives projected onto Listing's plane from behind the head (Fig. 3, left) and from its left side (Fig. 3, right). If we could present several targets at an equal distance from the eye and placed at $30^{\circ}$ intervals left and right of the fixation point (so that they always stimulate the horizontal meridian of the eye), these targets would form a full circle in space. In Fig. 3A, where the eye looks straight ahead at Listing's primary position, we view this circle edge-on from both perspectives. Symbols indicate the locations of targets that give $0^{\circ}$ (i.e., current fixation, $\mathbf{O}$ ), $30^{\circ}(\square), 60^{\circ}$ (吕), and $90^{\circ}(\bigcirc)$ horizontal RE (the latter corresponds approximately to the maximum of peripheral vision ). In this initial case, we can define the targets to be displaced horizontally relative to current gaze direction from either the oculocentric or craniotopic (head-fixed) perspectives. (Note: this specifies that the horizontal retinal meridian is that retinal arc intersected by the head-fixed horizontal plane containing the primary gaze direction when the eye is at primary position).

If we correctly adhere to these definitions, then the trivial 
A
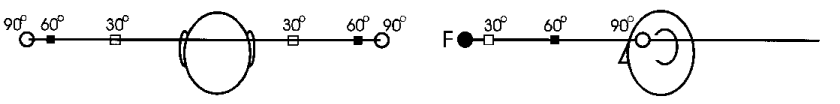

B

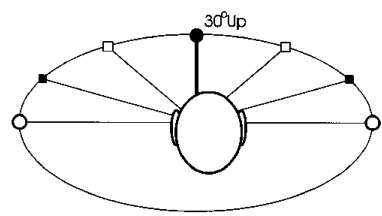

C

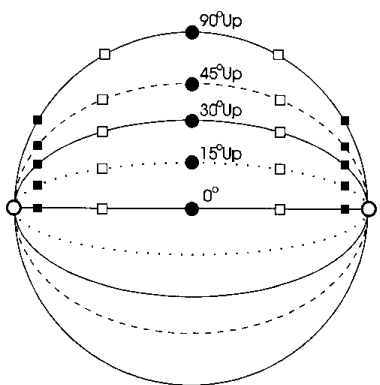

D

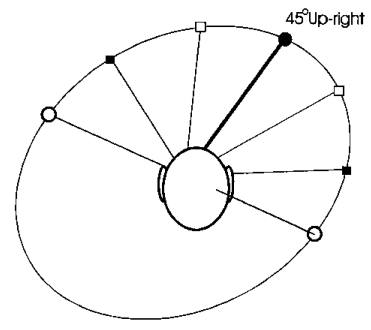

E
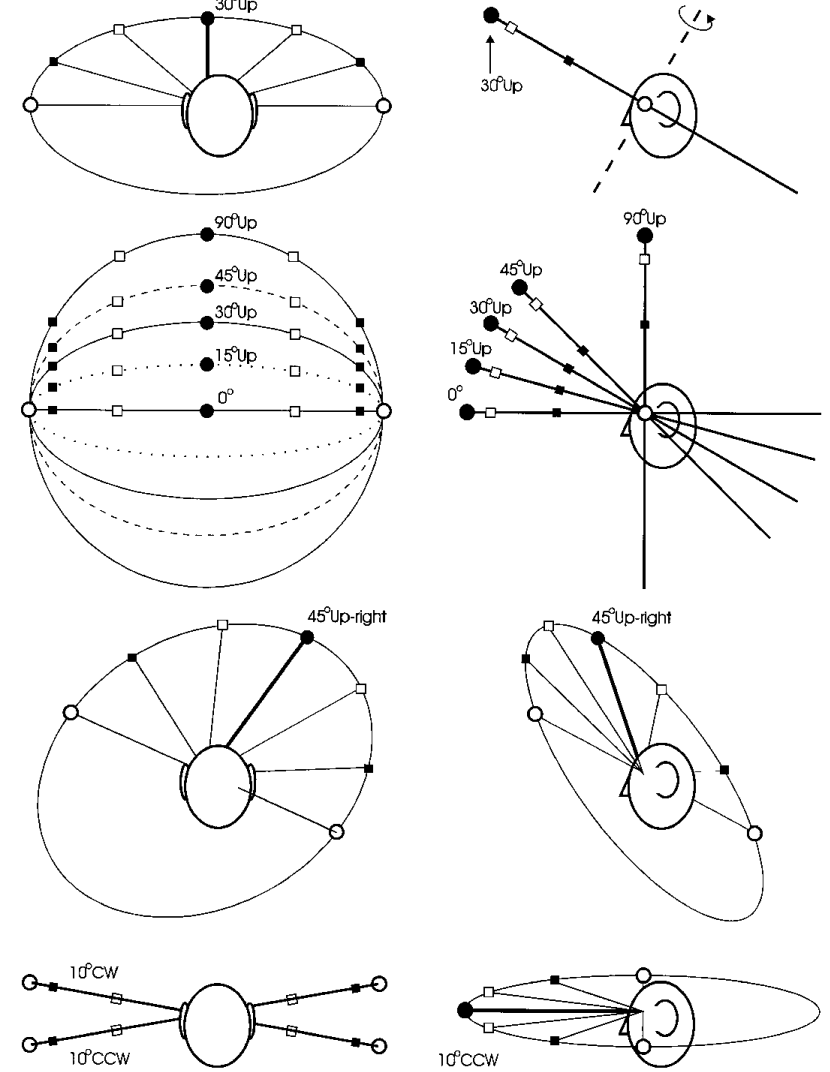

FIG. 3. Projections of targets on horizontal (relative to eye) circle centered on eye, viewed from behind head (left) and beside head (right). $\bullet$, tip of current gaze vector $(\mathrm{G}) ; \square, 30^{\circ}$ horizontal RE; $\mathbf{\square}, 60^{\circ}$ horizontal RE; , $90^{\circ}$ horizontal RE. $A$ : at primary position. $B: \mathrm{G}$ tilted $30^{\circ}$ up. --- , hypothetical axis of rotation for saccade. $C$ : $\mathrm{G}$ tilted upward by $0,15,30$, 45 , and $90^{\circ}$. $D: \mathrm{G}$ rotated $45^{\circ}$ obliquely up and to right. $E$ : eye is rotated $\pm 10^{\circ}$ clockwise $(\mathrm{CW})$ and counterclockwise $(\mathrm{CCW})$ about $\mathrm{G}$ from primary position.

relationship (Fig. 3A) between oculocentric and craniotopic horizontal displacement breaks down when gaze is displaced vertically. In Fig. $3 B$, current gaze direction has been rotated $30^{\circ}$ up. Thus, to maintain stimulation of the same retinal points, the circle of visual stimuli has also been rotated $30^{\circ}$ up (the spokelike vectors drawn from the eye to these targets in the back view help to visualize this tilt). Note that target displacements (from the back view), which by definition are still horizontal with respect to the eye, now require oblique gaze shifts with respect to the head. For example, $60^{\circ}$ leftward RE would now require an oblique leftward-downward eye movement. Figure $3 C$ more completely illustrates this effect for several elevations of gaze direction. Clearly this effect is increased by two factors: the vertical eccentricity of initial gaze direction from the primary position and the magnitude of the RE. At the illustrated extreme (obviously only in the range of eye + head gaze shifts), where gaze is elevated by $90^{\circ}$, an equally leftward and downward gaze shift would be required to satisfy $90^{\circ}$ leftward RE. Thus in 3-D the reference frame problem cannot be obviated by dealing solely with displacements.

Figure $3, D$ and $E$, illustrates two notable variations of this effect that will be simulated in more detail. First, should the retina be rotated torsionally about the line of sight, e.g., $10^{\circ}$ clockwise or counterclockwise as illustrated (Fig. $3 E$ ), the plane that stimulates horizontal RE (defined relative to the eye) will be oblique in the external world (Haustein and Mittelstaedt 1990), even while the eye is looking straight ahead. von Helmholtz (1925) was the first to demonstrate that Listing's law itself produces "false torsion" of the eye (i.e., torsion in Fick coordinates) at tertiary positions. Figure $3 D$ illustrates a tertiary gaze direction where our eye-fixed circle of targets has been tilted both vertically (as seen from the back view) and horizontally (as seen from the side view). Although we are not using Fick coordinates, small horizontal REs (near $\bullet$, in the range measured by von Helmholtz and followers) show the classic pattern of positiondependent tilt in the left-side projection (Fig. $3 D$ ). However, note that as horizontal RE increases leftward to $30^{\circ}$ and beyond, the projected direction of tilt reverses and becomes quite steep, leading to a strong horizontal (oculocentric)to-oblique (craniotopic) effect within a realistic oculomotor range. There are many other ways to project RE onto heador space-fixed coordinates (e.g., von Helmholtz 1925), but they will all distort the direction of RE as a function of eye position. Furthermore, as we shall see, the means illustrated (orthographic projection of desired oculocentric gaze directions onto Listing's plane) is particularly relevant to the physiology of generating head-fixed saccades.

From the perspective of saccade generation, there is one trivial solution to this problem that could, in theory, rescue the stimulus-response lookup table of the displacement model: the eye could rotate about the axis (e.g., Fig. $3 B$, $-{ }_{-}$) orthogonal to the plane containing current and desired gaze direction by an angle equal to that of RE. Gaze direction would thus sweep around the circle and accurately foveate the target in each case. This would essentially eliminate the reference frame problem by having both the sensory and motor aspects of visually triggered saccades operate in the same oculocentric reference frame. However, this would now require saccade velocity axes to tilt through an angle equal to the angle of eye position, for example tilting $30^{\circ}$ backward in Fig. $3 B$ compared with $A$. To see whether the oculomotor system uses this solution to its potential reference frame problem, we briefly review the geometry of 3-D saccade axes and plant mechanics.

\section{Axes of eye rotation during saccades}

Although Listing's law is usually visualized as a constraint on eye position, it implies an equally rigid constraint on the axes of eye rotation. Although it may seem paradoxical, the mathematics that govern rotations dictate that the axes of rotation for saccades must tilt torsionally out of Listing's plane to keep eye position vectors in Listing's plane (von Helmholtz 1925; Tweed and Vilis 1987). The general expression of this constraint is relatively complex (von Helmholtz 1925; Tweed and Vilis 1990a), so usually the simpler "half-angle rule" is cited. For example, for a horizontal saccade with gaze elevated $30^{\circ}$ above primary gaze direction, the axis of eye rotation must tilt backward from the vertical by $15^{\circ}$ out of Listing's plane, i.e., in the counterclockwise direction. This has been confirmed experimentally by com- 
puting angular eye velocity (i.e., instantaneous angular speed about the instantaneous axis of rotation) from eye position quaternions in humans and primates (Tweed and Vilis 1990a) and will be simulated in the next section.

\section{Eye muscle mechanics}

To control eye movements, the extraocular muscles must actively generate torques to compensate for two passively arising torques: one related to 3-D angular eye velocity as function of orbital tissue viscosity and one related to 3-D eye position as a function of orbital elasticity (Robinson 1975; Schnabolk and Raphan 1994; Tweed and Vilis 1987). Because the angular velocity-related torques must ideally tilt out of Listing's plane and the position-related torques must ideally stay in Listing's plane to give Listing's law (Tweed and Vilis 1990a), this poses a nontrivial problem for computing and matching these torques. Models that ignore this problem show considerable deviations from the ideal halfangle rule, leading to postsaccadic torsional drift (Schnabolk and Raphan 1994). It is now clear that this does not happen in real saccades ${ }^{1}$ (Straumann et al. 1995; Tweed et al. 1994), but we do not yet understand the physiological solution to this 3-D velocity-position problem.

In particular, we cannot understand this mechanism until we understand how eye muscle mechanics depend on eye position. For a time it was held that the direction of torque produced by each muscle is fixed with respect to the head (Miller and Robins 1987; Tweed and Vilis 1990a). However, recent anatomic studies suggest that the tissues near the ocular insertions of the muscles may exert a pulleylike effect (Demer et al. 1995), which could rotate the functional pulling directions of the eye muscles by anywhere from 0 to $100 \%$ with eye position. It has further been suggested that these pulleys may cause muscular torques to rotate by the correct amount to implement the half-angle rule, perhaps obviating the need for a neural implementation of Listing's law (Demer et al. 1995).

The idea of a muscular solution to Listing's law is theoretically appealing, but it must be emphasized that the halfangle rule pertains only to eye velocity, not eye position. As a result, this mechanical theory of Listing's law implies a level of mechanical complexity that cannot be addressed by the current data derived from static eye positions (e.g., Demer et al. 1995). For example, in many cases (including several simulated in the following text) the same muscles that generate the dynamic torsional torques during a saccade would also contribute to the zero-torsion position-related torque during and at the end of movement. If the positiondependent torsional torques in these muscles persisted at the end of the saccade, they would cause eye position to drift

\footnotetext{
${ }^{1}$ Minute and transient postsaccadic torsional drift does occur at the end of saccades in healthy subjects (Straumann et al. 1995; Tweed et al. 1994), but this can be modeled by simply underestimating the torsional component of plant elasticity in K (Fig. 1) (Tweed et al. 1994). We did not do this, because these transients do not affect final steady-state position (the focus of this investigation) and they are $\geq 1$ order of magnitude smaller than the effects that we did simulate. Furthermore, for the sake of simplicity we employed a "quasistatic" model of plant elasticity in this investigation, in which elastic forces on the eye are determined uniquely by the instantaneous rotational displacement of the eye relative to primary position independent of eye velocity history.
}

out of Listing's plane. Thus a purely muscular solution to the 3 -D velocity-position problem would require that muscular torques tilt torsionally as a function of eye position, but only in proportion to the eye velocity (specifically, the ratio between the muscle's contribution to velocity and position ). ${ }^{2}$ Again, there is absolutely no physiological evidence for such a mechanism at this time, but in light of the unexplored potential of muscular "pulleys" and the recent excitement about their possible role in Listing's law, we give this theory due consideration.

\section{Implications for a 3-D displacement model}

Armed with the above geometric constraints, we can now consider their implications for implementing the displacement model in 3-D. To approach this subject intuitively, we initially treat the displacement scheme as a fixed mapping from each point on the retina onto a unique pattern of eye muscle activation, i.e., leftward RE onto contraction of the lateral rectus muscle of the left eye (Fig. 4). To simplify matters, we assume that the lateral rectus muscle (-) rotates the eye about the vertical axis (_-) in Listing's plane (-- ) when the eye is at primary position (Fig. 4A) and ignore (for the moment) the contributions of other muscles. Second, Fig. 4 only considers the phasic contribution of the muscle (imagine the eye is suspended in a viscous medium with no position-dependent forces ). Finally, for the sake of clarity we exaggerate the range of eye movement, using the most extreme situation from Fig. $3 C$.

Figure 4 portrays the eye as viewed from the left side of the head, initially at the primary position (Fig. 4A, left). When a distant target (let us say the reader) appears to its left, the hypothetical retinal ganglion cells that specify $90^{\circ}$ leftward RE are stimulated. In this case appropriate activation of the lateral rectus muscle yields an accurate and kinematically correct saccade (Fig. 4A, right). No reference frame problem occurs, because the vertical axis of rotation required by Listing's law is orthogonal to the initial and final gaze directions. However, the situation becomes nontrivial when the horizontal saccade is generated from the $90^{\circ}$ upward initial position (Fig. 4, B-D, left). A distant leftward target again stimulates the same ganglion cells for $90^{\circ}$ leftward RE as in Fig. $4 A$ (e.g., compare Fig. 3, $A$ and $C$ ), as will be confirmed quantitatively. However, in this case mapping the $90^{\circ}$ leftward RE onto a contraction of the lateral rectus will not necessarily lead to foveation of the target. The precise outcome will clearly depend on the amount that pulling direction of the lateral rectus muscle rotates with eye

\footnotetext{
${ }^{2}$ These statements assume that plant viscosity does not depend systematically on eye position. If we relax this theoretical constraint, then the halfangle rule could arise from purely passive forces. For example, during horizontal saccades an asymmetric viscous drag on the lower and upper portion of the eye might arise from the asymmetry in stiffness between superior and inferior orbital tissues when the eye is elevated/depressed. This would tend to predict the transient torsional velocity components of the half-angle rule. The active muscular torque vectors could thus be constrained to Listing's plane without the need for position-dependent muscle mechanics. However, there is no real evidence for either idea. Moreover, this has no direct bearing on the current study other than the physical interpretation $R$ in our linear plant equations and the schematic illustration of muscle actions in Fig. 4. The linear plant equations, and thus the simulations, remain the same.
} 


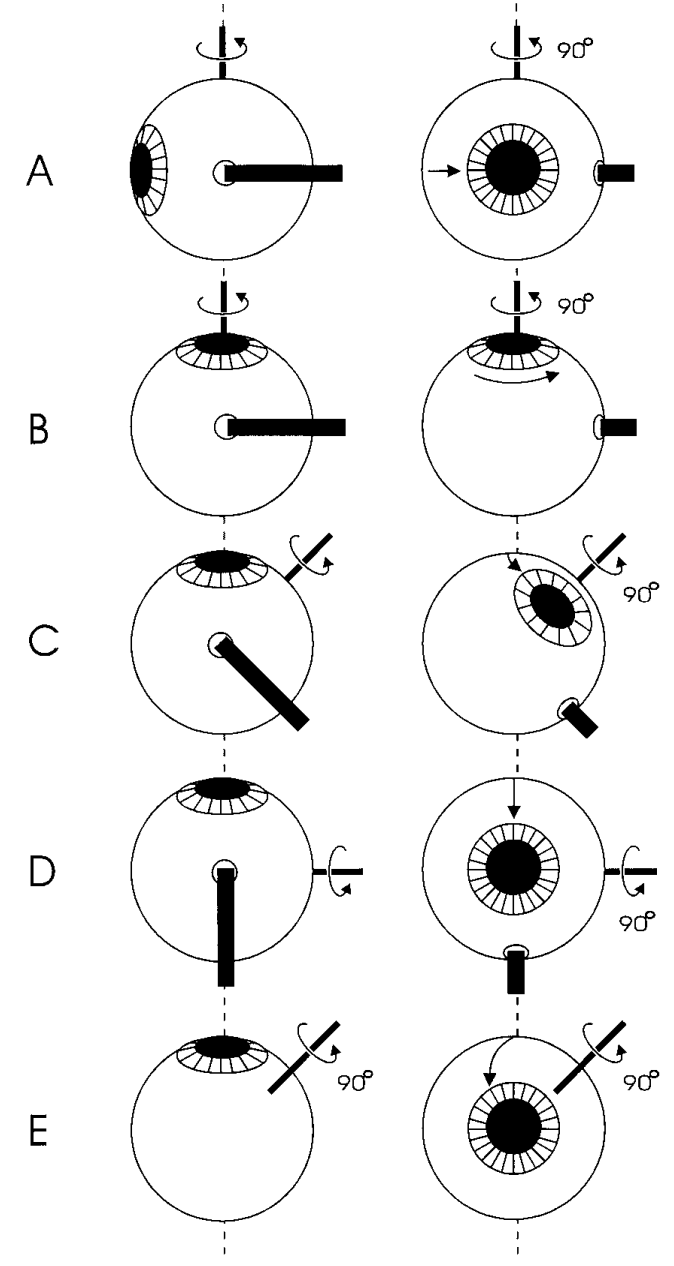

FIG. 4. Schematic illustration of displacement scheme with 0 , partial, and complete eye position dependence of eye muscle pulling directions. Left eye, idealized lateral rectus muscle (- $(-)$, and axis of rotation controlled by that muscle $(-)$ are viewed in craniotopic reference frame from perspective to left side of "subject's", head. Listing's plane $(---)$ is viewed edge-on. Left: initial positions (with muscle relaxed). Right: final positions (after eye has rotated $90^{\circ}$ about muscle's axis ). Initial RE is $90^{\circ}$ leftward in each case. $A$ : eye initially looks straight ahead. $B$ $D$ : initial eye position is rotated $90^{\circ}$ upward from straight ahead. $B$ : muscle pulling directions are independent of eye position. $C$ : muscle pulling directions are $50 \%$ dependent on eye position. $D$ : muscle pulling directions rotate completely with eye position. $E$ : correct response, which cannot be obtained by activating lateral rectus alone. In this case, axis of rotation tilts out of page, i.e., there is as much vertical rotation as horizontal rotation.

position. Because this remains unclear (Demer et al. 1995), we consider three representative possibilities.

Suppose first (Fig. $4 B$ ) that the action of the lateral rectus is fixed in the head (a craniotopic plant model). With the eye looking up, the trivial visuomotor mapping would now spin the eye about the visual axis without changing gaze direction, obviously failing to acquire the target (which is directed out of the page). We could then imagine that the oculomotor system has solved this problem by evolving eye muscles whose pulling directions remained fixed relative to the eye (an oculocentric plant model). As in the illustrated example (Fig. $4 D$ ), this would produce accurate foveation of the target. However, in tilting the pulling direction of the muscle $100 \%$ along with the eye, we now have a rotation about the head-fixed torsional axis, which would produce a large counterclockwise violation of Listing's law. Only with the intermediate $50 \%$ eye position dependency (Fig. $4 \mathrm{C}$ ) will the correct axis of rotation for Listing's law be implemented (Tweed and Vilis 1990a). Unfortunately, now gaze direction sweeps about this backward tilting axis to an upward-leftward position, producing an error intermediate in magnitude between the craniotopic and oculocentric models. This argument suggests that at a very basic geometric level a fixed visuomotor mapping must choose between Listing's law and saccade accuracy: it cannot have both.

Note that Fig. 4 does not suggest that there is no kinematically correct solution to this problem, but rather that a solution cannot be reached within the constraints of a realistic displacement scheme. In particular, $90^{\circ}$ horizontal RE and Listing's law can be satisfied from an eye position elevated by $90^{\circ}$ if the eye rotates not only horizontally and torsionally but also vertically, i.e., about an axis with a large horizontal component (Fig. $4 E$ ). However, none of the fixed visuomotor schemes illustrated in Fig. 4, $B-D$, can provide this component. To make such a movement with the horizontal rectus alone, one would have to propose that this muscle produces a strong phasic downward torque (in craniotopic coordinates) as a function of upward eye position, perhaps slipping under the eye when it looks up. Furthermore, because the final position has no vertical component, the initial static upward torques from the elevator muscles would have to simultaneously disappear without any change in their neural input! This is clearly at odds with the current understanding of the oculomotor system, being unrealistic from mechanical, physiological, theoretical, and evolutionary points of view (e.g., Demer et al. 1995; Hepp and Henn 1985). Therefore we conclude that Fig. $4 E$ cannot be produced by the same pattern of muscle activation that was used in Fig. $4 A$, even though both movements provide the correct response to the same RE. This suggests that different combinations of horizontal and vertical eye muscles must be activated to satisfy the same RE, depending on initial eye position.

These arguments help to establish an intuitive framework for several computational problems but fail to demonstrate these problems with mathematical rigor, quantify them in a realistic behavioral context, or offer possible physiological solutions. To these ends, formal computational models were required. In developing these, our first goal was to identify the computations necessary for the best possible 3-D version of the displacement-feedback model. Because it would seem oxymoronic to follow a 3-D scheme that cannot obey Listing's law (e.g., Fig. $4, B$ and $D$ ), we chose to pursue the basic scheme illustrated in Fig. 4C. To evaluate the hypothesis that the eye muscles can solve the kinematic problems associated with Listing's law (Demer et al. 1995; Schnabolk and Raphan 1994), we equipped this model with the ideal theoretical plant to perfectly implement the half-angle rule. We then evaluated the hypothesis (Fig. $4 C$ ) that a half-angle rule displacement model would sacrifice saccade accuracy as some function of eye position, and we evaluated whether, if such were the case, it would result in significant behavioral problems within a realistic oculomotor range. Our subsequent goals were to identify the additional neural computations necessary for the ideal behavioral solution (e.g., Fig. $4 E$ ), incorporate these into our model, and evaluate their 

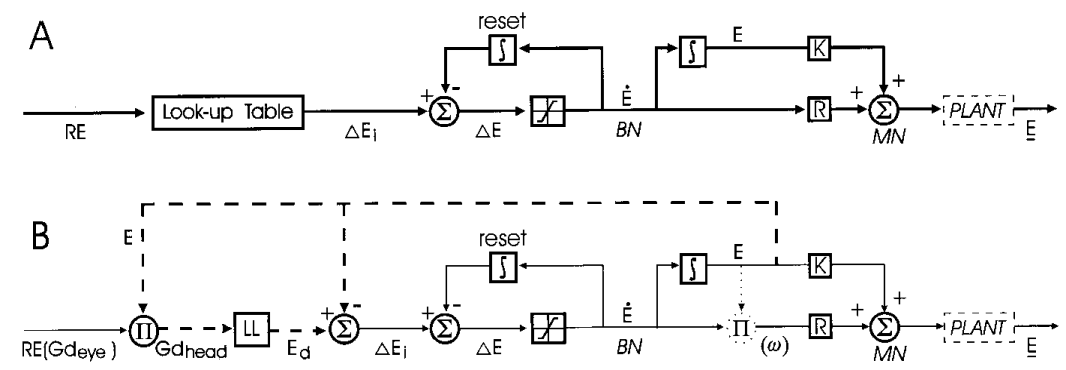

FIG. 5. A: 3-D displacement-feedback model. In this model components of RE were mapped directly onto same-scale components of $\Delta E_{\mathrm{i}}$ (this mapping process is designated as "lookup table"). During saccade, displacement feedback from resettable integrator $\left(\int\right)$ was subtracted from $\Delta E_{\mathrm{i}}$ to compute instantaneous 3-D ME $(\Delta E)$ that drives burst neurons. Outputs of this feedback loop are torsional, vertical, and horizontal components of rate of position change $(\dot{E})$. Because $\dot{E}$ is derivative of position with respect to time, its components were input directly into 3 integrators $\left(\int\right)$ that generate torsional, vertical, and horizontal components of $E$. $\dot{E}$ and $E$ vectors were left multiplied by plant viscosity (R) and elasticity (K) matrix constants, respectively, before summing componentwise at motoneurons. $E$, actual eye position. In simulations illustrated below, this model was equipped with "linear plant" model, which reduced need for position-dependent computations upstream. $B$ : new spatial model for generation of saccades in 3-D. This model was based on displacement-feedback model, but incorporated additional position-dependent transformations $(---)$. RE is represented as vertical and horizontal components of desired gaze direction relative to eye $\left(G d_{\text {eye }}\right)$. $G d_{\text {eye }}$ was then rotated multiplicatively ( $\Pi$ ) by 3-D eye position $(E)$ into craniotopic reference frame $\left(G d_{\text {head }}\right)$. Here $E$ was derived from output of downstream neural integrator, but it could also be derived from sensory organs in eye muscles. Desired 3-D eye position $\left(E_{\mathrm{d}}\right)$ in Listing's plane was then computed from $G d_{\text {head }}$ with the use of an operation (LL) first described by Tweed and Vilis (1990b). Subtracting $E$ from $E_{\mathrm{d}}$ yielded initial desired change in eye position $\Delta E_{\mathrm{i}}$, which completed visuomotor transformation. With craniotopic plant model (Crawford and Vilis 1991; Tweed and Vilis 1987), $E$ was divided by copy of $E(\cdot \cdots)$ to produce 3-D angular velocity command $(\omega)$. With linear plant model, latter step was unnecessary. (Vector symbols are italicized in text.)

biological significance by testing the resulting model against the 3-D displacement model under realistic behavioral conditions ( see SIMULATIONS).

\section{MODELS}

This section describes the theoretical development of our models, focusing on the physiological constraints that led to their specific algorithms (Fig. 5). The detailed math pertaining to the implementation of these algorithms, computation of RE from objective target directions and 3-D eye position (Fig. 3), and 3-D rotational kinematics of the eye are described in the APPENDIX.

\section{Modeling the 3-D plant and its control signals}

Recent experiments have shown that the signals within the oculomotor velocity-to-position integrator and reticular formation burst neurons are organized in a 3-D, head-fixed, musclelike coordinate system that seems to align with Listing's plane (Crawford 1994; Crawford and Vilis 1992; Henn et al. 1989). However, these experiments do not fully specify the nature of the 3-D signal coded within these coordinates. To understand the possible options, one needs to briefly consider the math of rotational kinematics. The nontrivial relationship between eye positions and velocities during saccades is a reflection of the general multiplicative relationship between angular velocity $(\omega)$, the 3-D angular position vector $(E)$, and its derivative with respect to time $(\dot{E})$, which is expressed with the use of quaternion algebra as

$$
\dot{E}=\omega E / 2
$$

Tweed and Vilis (1987) were the first to describe this relationship in detail and to demonstrate the potential problem that it poses for the neural integrator theory: in 3-D, eye position is not the integral of $\omega$. In concrete terms, integrat- ing the torsional components of $\omega$ necessary for Listing's law would yield incorrect torsional position signals relative to Listing's plane. Therefore it was suggested that the velocity-to-position transformation for saccades incorporates $E q$. 1 , in effect multiplying the $\omega$ signal (assumed to be encoded by burst neurons) by a feedback copy of $E$ to yield $\dot{E}$ ( $E q$. $1)$, which then could be integrated to yield a correct eye position command (Tweed and Vilis 1987).

This model was mathematically and behaviorally correct (Tweed and Vilis 1990a; Tweed et al. 1994), but some of its physiological assumptions may not be correct. In particular, recent evidence suggests that burst neurons encode something closer to $\dot{E}$ than $\omega$. In Listing's coordinates, a burst neuron vector coding $\dot{E}$ would (unlike $\omega$ ) always code zero torsion when Listing's law is obeyed. Consistent with this, the activity of torsionally tuned burst neurons does not correlate well with the measured torsional components of $\omega$ during saccades in Listing's plane (Hepp et al. 1994). Second, lesioning the midbrain torsional burst neurons does not cause the planar range of eye positions to break down (Suzuki et al. 1995), as it should if these neurons encode the torsional axis tilts seen in $\omega$. Finally, the velocity-to-position model of Tweed and Vilis (1987) predicted that damage to the vertical integrator would also affect horizontal position holding, but this does not occur in real data (Crawford 1994). To be consistent with these data, we modeled burst neurons as coding $3-\mathrm{D} \dot{E}$ in a coordinate system aligned with the head-fixed Listing's plane (Crawford and Vilis 1992) and input this directly into a 3-D integrator to compute the eye position control signal (Fig. 5A).

Because burst neurons coding $\dot{E}$ would not specify the torsional components in actual eye velocity, this requires the half-angle rule to be implemented downstream. For our displacement model (Fig. 5A), we chose to implement this process in the plant itself (Fig. $4 C$ ). Although this plant might be mechanically complex (as discussed previously), 
it was modeled very simply by removing the $\omega$ term from the motoneuron transfer function

$$
M N=K \underline{E}+R \underline{E}
$$

where $M N$ is mean motoneuron firing rate, $\underline{E}$ and $\dot{E}$ are the 3-D craniotopic vectors or quaternions of actual eye kinematics, and matrix $K$ represents plant elasticity and $R$ represents the equivalent to plant viscosity, expressed in $\dot{E}$ coordinates. The nonlinearity in Eq. 1 thus only exists implicitly in $E q .2$, in the position-dependent multiplicative relationship between explicit $\dot{E}$ and implicit $\omega$. Equation 2 has been called the "linear plant model" for its resemblance to similar 1-D models ( Tweed et al. 1994). From this equation it should be clear why these motoneurons require internal representations of $E$ and $\dot{E}$ as input and (if modeled in Listing's coordinates ) that Listing's law should hold as long as these input vectors code zero torsion.

\section{Implementing the displacement-feedback model in 3-D}

In both the displacement and spatial models of 1-D saccade generation (Fig. 1), burst neurons are driven by a displacement command called ME. The geometry of such a displacement command is relatively trivial in 2-D, but in 3$\mathrm{D}$ we must choose among several possible interpretations. For example, Tweed and Vilis (1991b) modeled ME as encoding a head-fixed axis of rotation (Fig. $4 B$ ), which was appropriate to control burst neurons that code $\omega$. However, if burst neurons encode $\dot{E}$, then a different interpretation of $\mathrm{ME}$ is specified. In the displacement-feedback loop (Jürgens et al. 1981; Fig. 1B), ME equals the total integral of burst neuron activity over each saccade [ the same applies to the computationally similar Scudder (1988) version of the displacement hypothesis]. In 3-D, this must be done for each component of $\dot{E}$. The result is not a rotation, but rather a vector describing $3-\mathrm{D}$ change in eye position $(\Delta E)$. This can be further defined as the vector difference between initial and final 3-D eye position (Van Opstal et al. 1991)

$$
\Delta E=E_{\mathrm{f}}-E_{\mathrm{i}}
$$

Thus the initial ME command for our displacement-feedback loop was a desired change in eye position vector (Fig. $5 A)$. This is consistent with the experimental finding that stimulation of a given site in the deeper motor layers of the superior colliculus produces a constant $\Delta E$ in Listing's plane independent of initial eye position (Hepp et al. 1993; Van Opstal et al. 1991). For reasons that we discuss further, Van Opstal et al. (1991) chose to tentatively interpret $\Delta E$ as physiological RE (more precisely, their stimulation data did not distinguish between $\mathrm{RE}$ and $\Delta E$ ). This suggests that visual RE could be mapped trivially onto corresponding zero-torsion $\mathrm{ME}$ commands (for $\Delta E$ in Listing's plane).

This is precisely the scheme that we chose as the optimal 3-D formulation of the displacement-feedback model. This process is labeled as a lookup table in Fig. $5 \mathrm{~A}$ to reflect the possibility that this represents a site-to-site projection from sensory to motor "maps" in the brain. As we demonstrate, this model (equipped with the linear plant) essentially implements the visual-muscular mapping illustrated in Fig. $4 C$. However, the success of this model relies on the assumption that $\mathrm{RE}$ and $\mathrm{ME}$ (modeled as $\Delta E$ ) are equal.

\section{$R E$ and $M E$ are not geometrically equivalent}

In their 3-D description of the superior colliculus, Van Opstal et al. (1991) interpreted $\Delta E$ vectors as RE largely as a matter of convenience, because the characteristics of 3$\mathrm{D} \Delta E$ had not been thoroughly discussed in the oculomotor literature. However, they also noted briefly (see APPENDIX) (Hepp et al. 1993) that mathematically these two vectors are in fact only similar within a restricted range of eye positions in Listing's plane. Here we further pursue the difference between these two vector classes. Understanding the difference between these displacement codes is crucial to our thesis, because it provides the computational language necessary to describe the internal analogues of the visuomotor problems described intuitively in the BACKGROUND section, and thus their solutions.

The difference between RE and ME was not evident in 2-D models because both were represented in the same way, i.e., much like the vector representation of a 2-D translation. This equivalence has led to the convention of referring to displacement codes as "oculocentric' and position codes as "'craniotopic.' However, these conventions fall apart in 3-D, where displacements can be represented in either reference frame. We have already seen two types of 3-D displacement defined in craniotopic coordinates: the fixed-axis rotation used by Tweed and Vilis (1990a) to model ME and $\Delta E$. Equation 3 dictates that $\Delta E$ is defined in the same 3-D, craniotopic coordinate system as the representations of eye position from which it is derived. In our model, ME inherits these characteristics from its output by mapping directly onto downstream transformations that exist entirely in 3-D, craniotopic coordinates. Therefore (as will be confirmed) this vector specifies a displacement that is fixed with respect to the head, independent of eye position, and has some finite torsional component (even if this is 0 ). In contrast, RE is defined by its input to be oculocentric and 2-D, i.e., there is no such thing as "'torsional RE.',

This difference is neither arbitrary nor purely theoretical. By definition, the transformation from 2-D oculocentric RE and 3-D craniotopic ME should be the point at which the input-defined sensory code is converted into a motor code. However, no provision for a position-dependent reference frame transformation could be incorporated into this stage of our displacement model, because this would violate the basic premise of the displacement hypothesis. Furthermore, the degrees of freedom problem between RE and ME was addressed trivially, by setting torsional ME to equal zero in Listing's coordinates. Thus, if our displacement model failed to correctly implement Listing's law or to solve the reference frame problem demonstrated in Fig. 3, this is where the internal analogue of the problem would reside.

We have already suggested that this scheme will show reference-frame-related errors, but there is also reason to believe that its trivial 2-D-to-3-D transformation will only work in highly idealized head-fixed conditions. Recent reports have shown that under more natural head-free conditions, Listing's plane shifts, tilts, and is marked by a perpetual series of systematic torsional violations and corrections (Crawford and Vilis 1995; Tweed et al. 1995). To demonstrate this problem, we only need to simulate a simple situation encountered during passive rotations of the head. Slow 
phases of the vestibuloocular reflex routinely drive eye position torsionally, even when the axis of head rotation aligns with Listing's plane (Crawford and Vilis 1991). In the real data, any remaining ocular torsion at the end of a head movement is then corrected by the first saccade (Crawford and Vilis 1991), suggesting that these saccades have a torsional goal in Listing's plane.

Thus $\Delta E$ (or any other 3-D measure of ME) must routinely have finite torsional components to correct or anticipate slow-phase-dependent violations of Listing's law when the head is free, requiring continuous comparisons between current and desired 3-D eye position. Furthermore, this craniotopic torsional component of ME could not be computed independently from gaze without disrupting accuracy (because craniotopic torsion contributes to gaze direction when the eye is not at primary position). The model of Tweed and Vilis (1990b) possessed a Listing's law operator that provided a correct 2-D-to-3-D transformation for any desired gaze direction and initial eye position, but this important feature has been largely overlooked because other aspects of that model were contradicted (Van Opstal et al. 1991). Our strategy was to incorporate a similar 2-D-to3-D transformation into our model in a way that did not contradict the known physiology.

\section{New model of the visual-motor transformation}

To deal effectively with the reference frame and degrees of freedom problems identified in the preceding text, we were forced to step outside the constraints of the displacement hypothesis. The geometric and physiological constraints described defined not only which visuomotor operations were required (a reference frame transformation and a 2-D-to-3-D transformation with comparisons with current eye position) but also their specific order. Working our way upstream from ME: first, the need for a 3-D ME signal that corrects torsion relative to positions on Listing's plane called for an internal comparison between initial and desired 3-D eye position. Second, this desired eye position on the head-fixed Listing's plane had to be constructed from 2-D visual signals, requiring a Listing's law operator (Tweed and Vilis 1990b). Finally, because the latter computations had to be performed in the craniotopic reference frame (i.e., eye position cannot be defined relative to the eye), the visual information was first put through a reference frame transformation. Rather than develop an entirely new model, we simply incorporated these features into the visual-motor transformation of our displacement model. The result was a hybrid spatial displacement-feedback model (Fig. $5 B$ ), but for brevity we refer to this as the 3-D spatial model.

Stepping outside of the bounds of a traditional displacement scheme also allowed us to test other internal positiondependent transformations in the neuromuscular control system. For example, the mechanically simple craniotopic plant (Fig. $4 B$ ) takes in $\omega$ rather than $\dot{E}$ (Tweed and Vilis 1987). To control this plant, a burst neuron signal coding $\dot{E}$ would have to be multiplied by $E$ (perhaps presynaptically at motoneurons) to give $\omega$, thus implementing the half-angle rule at a very late neural stage (Fig. $5 B, \cdot \cdots$ ). [Conversely, with the oculocentric plant model (Fig. $4 D$ ), $\sim 50 \%$ of the phasic position-dependent torque in the plant would have to
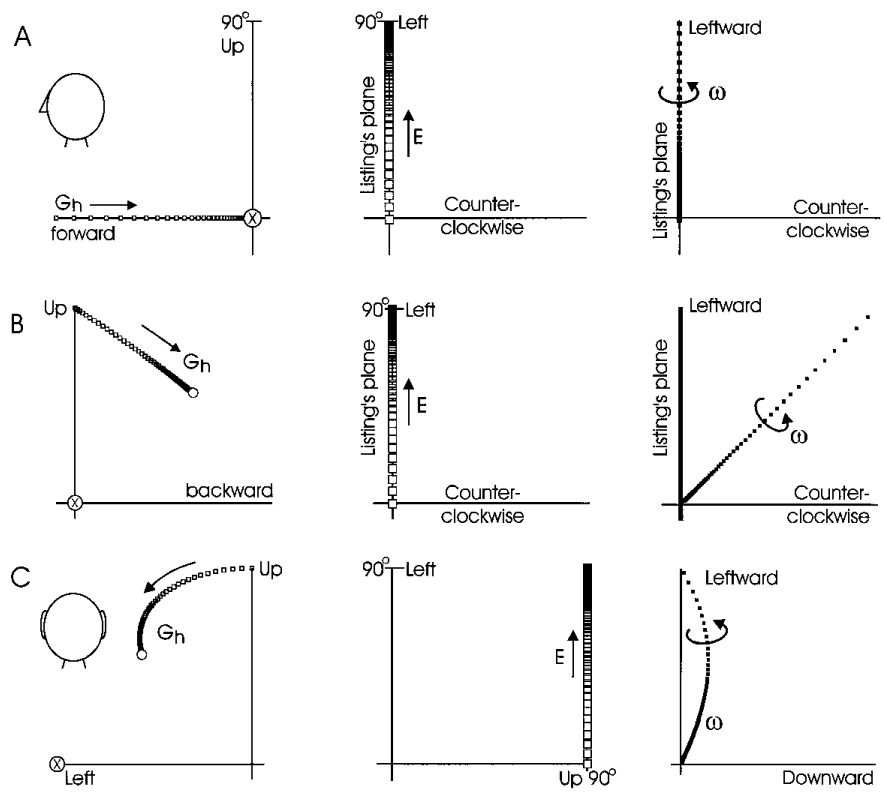

FIG. 6. Simulation of hypothetical situation from Fig. 4, generated by 3-D displacement model. Simulated data (a) are plotted in craniotopic coordinates. Left: 2-D projections of tip of constant-length vector parallel to current gaze direction (G). Desired gaze direction: (X). Middle: 2-D projection of tip of eye position vector. Right: angular velocity vectors (parallel to instantaneous axis of rotation.) $A$ : correct response to $90^{\circ}$ leftward RE from primary position. Vertical and horizontal components of simulated data are projected onto torsional-vertical plane orthogonal to Listing's plane as viewed from left side of head (see drawing). $B$ : incorrect response to $90^{\circ}$ leftward $\mathrm{RE}$ from initial position rotated $90^{\circ}$ upward from primary position, viewing simulated data from side. $C$ : same data as in $B$, but now viewing data vectors as they would project onto Listing's plane viewed from behind head.

be neurally "undone" in the burst neuron input to give the half-angle rule.] Indeed, a burst neuron signal coding $\dot{E}$ could be compensated downstream to control any plant model so long as the overall downstream transfer function remains constant. Similarly, this should completely specify the overall visuomotor transformations between RE and burst neurons independent of plant characteristics. To demonstrate these ideas, we used both the linear and craniotopic plant models in our spatial model. (Unless otherwise specified, the craniotopic plant configuration of the spatial model was used in the simulations.)

\section{SIMULA TIONS}

To clearly establish the difference between our spatial and displacement models, we began by examining their performance in the exaggerated position ranges described in the BACKGROUND section. Figure 6 illustrates the performance of the 3-D displacement model in the situation portrayed schematically in Fig. 4. This figure also serves to graphically introduce simultaneously evolving gaze directions, eye positions, and instantaneous angular eye velocities. Gaze direction relative to the head $\left(G_{\mathrm{h}}\right.$, Fig. 6 , left $)$ is illustrated by the tip of a unit vector that originates at the center of the eye and is parallel to the visual axis. Eye position ( $E$, Fig. 6, middle) is illustrated as the tips of vectors that extend from the origin in parallel to the axis of rotation relative to primary position (according to a 
right-hand rule), with length equal to the angle of rotation. Eye velocity ( $\omega$, Fig. 6, right) is also plotted in a similar right-handed coordinate system, but shows the instantaneous axis of rotation, with length equal to angular speed of rotation. The top two rows show the head-fixed torsional and vertical coordinate axes for these data as they would be viewed from the left side and projected onto the sagittal plane (as in Fig. 4, $A$ and $C$ ).

In Fig. 6A, left, the eye was initialized at primary position with gaze straight forward. A $90^{\circ}$ leftward target direction (i.e., straight out of the page as in Fig. 4) was input (X), trivially "stimulating", $90^{\circ}$ leftward RE. This caused the simulated gaze (left) to sweep leftward until it was parallel to the horizontal axis (i.e., pointing out of the page). Note that the position vector (middle) grew upward along the vertical axis and that instantaneous angular velocity (right) indicates a purely vertical axis of rotation. This is the trivial case simulated in 1-D models, where horizontal RE maps onto a horizontal change in eye position and purely horizontal velocity (as in Fig. $4 A$ ).

Figure $6, B$ and $C$, illustrates the performance of the displacement model where gaze direction (left) was initially straight up, parallel to the vertical axis. The target direction was again selected to be due left in craniotopic coordinates (X). Oculocentric RE was then computed by rotating this craniotopic direction vector by the inverse of initial eye position (see APPENDIX). As demonstrated qualitatively (Figs. 3 and 4 ), this target direction stimulated the same $90^{\circ}$ leftward (in oculocentric coordinates) RE from this eye position as in Fig. 6A. The kinematic response to this input is viewed from the side perspective in Fig. $6 B$ and from the behind perspective (along the vertical and horizontal coordinate axes) in Fig. 6C. Viewing the position trajectory (middle) from both perspectives, it is clear that the displacement model has again mapped $90^{\circ} \mathrm{RE}$ onto a $90^{\circ}$ leftward change in eye position (as illustrated by position vectors growing straight upward) independent of the initial vertical eye position (along the horizontal axis in Fig. $6 \mathrm{C}$ ). In terms of Listing's law, this posed no problem: eye position vectors remained in Listing's plane (Fig. 6B, middle) and the required concomitant velocity axis tilt was also observed (Fig. $6 \mathrm{~B}$, right). However, instead of sweeping toward the target ( $\mathrm{X}$, which again is straight out of the page), gaze direction (Fig. 6B, left) sweeps toward an extreme upward-leftward direction almost out the back of the head, missing the correct desired gaze direction by $55.8^{\circ}$. [Indeed, other than a slight curvature in the back view of the velocity trajectory (Fig. 6C, right), the displacement model showed the same kinematics illustrated schematically in Fig. 4C.] Thus the same fixed visuomotor mapping that worked correctly from primary position (Fig. 6A) failed to acquire the target from an elevated eye position (Fig. $6, B$ and $C$.)

Figure 7 shows the performance of the 3-D spatial model for the same tasks as in Fig. 6. From primary position, the response of this model to $90^{\circ}$ leftward RE (Fig. 7A) was indistinguishable from that of the displacement model (Fig. $6 A)$. However, the response was very different when eye position was elevated by $90^{\circ}$. As seen in the side view (Fig. $7 B$ ), eye position vectors were again confined to Listing's plane whereas the saccade axes tilted outward. However, this time, $90^{\circ}$ leftward RE caused the gaze vector to (cor- rectly) sweep downward to finally align with the horizontal axis (Fig. 7, left). Viewing the position trajectory from behind the head (Fig. $7 C$ ) it is evident that the $90^{\circ}$ leftward RE was now mapped onto a zero-torsion oblique change in eye position, causing gaze (Fig. 7, left) to sweep both leftward and downward, correctly acquiring the target. [In terms of the axes of rotation (Fig. 7, right), this is the same solution shown schematically in Fig. 4 E.] Thus the same RE ideally mapped onto two very different gaze shifts in Fig. 7, $A$ and Fig. 7, $B$ and $C$. By taking initial 3-D eye position into account, the $3-\mathrm{D}$ spatial model was able to generate the correct kinematics even in these most extreme cases.

\section{Toward a behavioral test in the oculomotor range}

3-D KINEMATICS. We next compared the performance of the 3-D spatial and displacement models for eye-in-head saccades within a more realistic oculomotor range of $\mp 50^{\circ}$. Our first goal was to evaluate the plausibility of the idea that Listing's law is implemented mechanically. Figure 8 shows simulated eye position $(E)$ and eye velocity $(\omega)$ trajectories for leftward saccades between horizontally displaced targets at seven vertical levels. In each case, eye position was initialized in Listing's plane, with gaze direction $30^{\circ}$ to the right and at vertical levels (through $15^{\circ}$ intervals) from $45^{\circ}$ below to $45^{\circ}$ above primary position. For each of these initial positions, RE was computed from a simulated target "placed", symmetrically on the opposite horizontal side (i.e., due left in space) and was then used as input to both the 3-D spatial model (Fig. 8A) and the displacement model (Fig. 8B). Figure 8 , left, shows torsional eye position plotted against vertical position (i.e., showing $7 E$ trajectories in Listing's plane as viewed from above the head) and Fig. 8, middle,
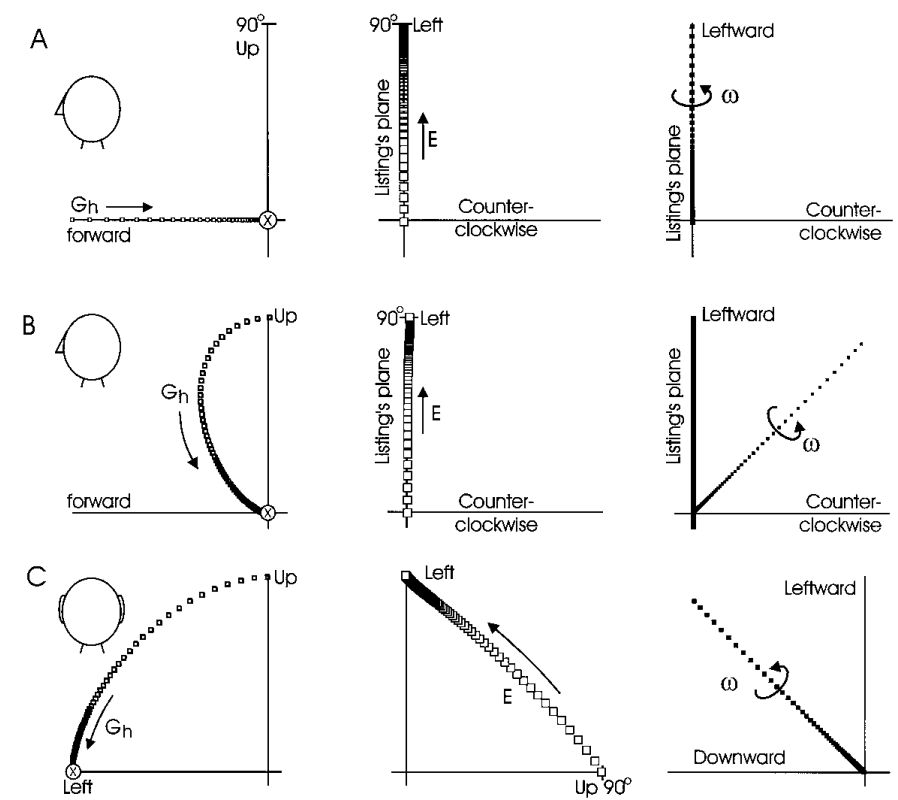

FIG. 7. Simulation with 3-D spatial model with the use of same inputs and figure conventions as in Fig. 6. A : response to $90^{\circ}$ leftward RE from primary position; data viewed from side of head-fixed coordinate system. $B$ : response to $90^{\circ}$ leftward RE from initial position rotated $90^{\circ}$ upward from primary position, viewing data from side of head. $C$ : same data as in $B$ now viewed from perspective behind head. 
(E)
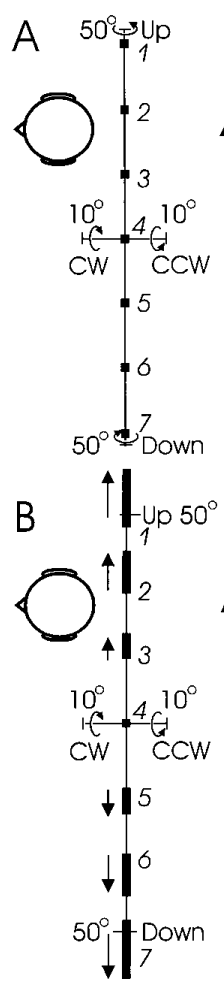

(E)

$(\omega)$

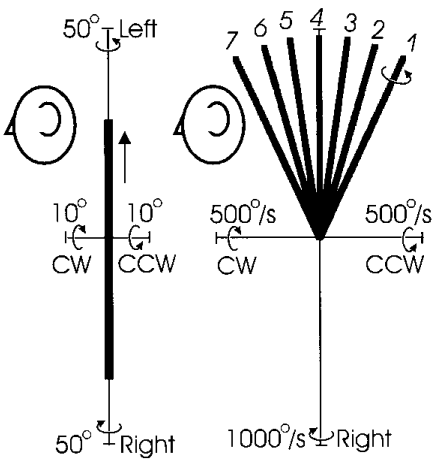

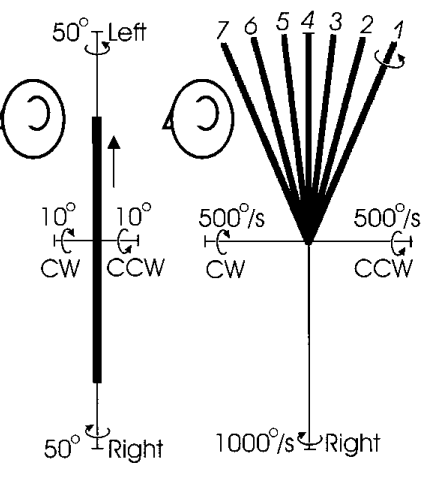

FIG. 8. Comparison of kinematics of Listing's law simulated by 3-D spatial model $(A)$ and 3-D displacement model $(B)$. In each case, eye position was initialized in Listing's plane with gaze $30^{\circ}$ right and at 7 vertical levels from $45^{\circ}$ down to $45^{\circ}$ up, through $15^{\circ}$ intervals (labeled $1-$ 7 ), and target directions $60^{\circ}$ due left (in craniotopic coordinates) were input for each of these positions. Left: top view of simulated 3-D eye position vectors $(E)$, showing vertical components in 0 torsion plane. Middle: side view of same data, showing overlapping horizontal components of $E$ in 0 torsion plane for the 7 movements. Right: side view showing torsional tilts of angular velocity vectors $(\omega)$ for same simulated saccades (1-7). The only important difference between $A$ and $B$ is presence of unusual outward-going vertical $E$ components in $B$, but this did not constitute violation of Listing's law and is explained in Fig. 10.

plots torsion against horizontal position (i.e., viewing Listing's plane from beside the head, where the mainly horizontal $E$ trajectories overlap). Figure 8, right, shows the torsional and horizontal (i.e., about the vertical axis) components of angular velocity $(\omega)$ as they would be viewed from beside the head (horizontal vs. vertical plots are provided below). Corresponding eye position trajectories and velocity axes are labeled 1-7.

Both models performed equally well at maintaining eye position in Listing's plane and, concomitantly, generating the required velocity axis tilts out of Listing's plane (by essentially half the angle of gaze elevation, as evident by comparing Fig. 8, left and right). Moreover, switching the plants between these models had no noticeable effect on Listing's law (as long as the internal computation of the half-angle rule was removed from the spatial model and added into the direct path of the displacement model). Thus the "neural" implementation of these tilts (see APPENDIX, Eq. 17; used in Fig. 8A) and the hypothetical "muscular", implementation (see APPENDIX, Eq. 9-11; used in Fig. 8B) generated indistinguishable behavior, in terms of Listing's law, so long as eye position was initialized in Listing's plane.

This ambivalence disappeared when initial eye position had a torsional component outside of Listing's plane. Figure 9 shows 3-D eye position vectors during simulated saccades evoked from an initial $10^{\circ}$ clockwise torsional eye position as observed following passive head rotations (Crawford and Vilis 1991) or induced experimentally by stimulation of the midbrain (e.g., Crawford and Vilis 1992). A simulated $30^{\circ}$ upward (relative to the head) visual target was used as input to both the 3-D spatial model ( $\mathbf{\square}$ ) and the 3-D displacement model $(\square)$. The displacement model mapped components of the resulting RE onto a position change vector parallel to Listing's plane (Fig. 9A), thus failing to correct the torsion. In contrast, the spatial model used both RE and eye position information to compute the unique 3-D ME command that both corrected the initial torsion and (as we shall see) accurately acquired the visual target.

ACCURACY OF FINAL GAZE DIRECTION. Most investigators would agree that regulation of ocular torsion is of only secondary importance compared with accurate gaze control. However, if we examine the "back view" (Fig. 9B) of the vertical versus horizontal position trajectories of the previous simulation, it is evident that torsion also poses a unique problem for saccade accuracy. Whereas the position vectors generated by the spatial model (ם) followed a purely upward trajectory (i.e., the position vectors grow rightward on the page) toward the upward-displaced target, the trajectory generated by the displacement model $(\square)$ tilted obliquely, following a line rotated $10^{\circ}$ counterclockwise from the correct trajectory. The reason for this error was a special instance of the oculomotor reference frame problem: because the simulated retinal map was initially rotated $10^{\circ}$ from its
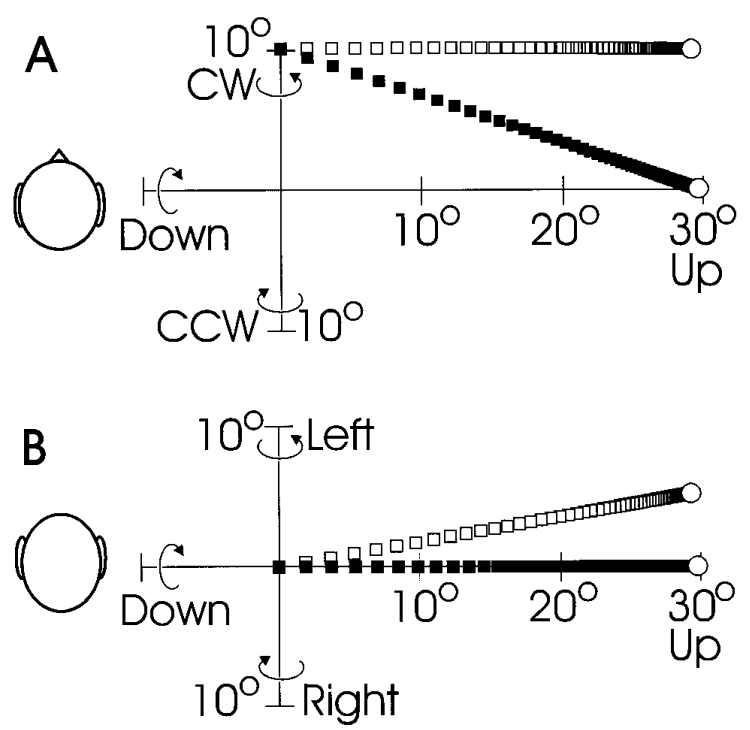

FIG. 9. Performance of 3-D spatial ( $\square$ ) and 3-D displacement ( $\square$ ) models in presence of initial torsional deviation from Listing's plane. Simulated 3 -D position vectors are shown for eye movements elicited by target located $30^{\circ}$ upward (in craniotopic coordinates) from initial eye position at $10^{\circ}$ clockwise torsion. $A$ : top view of vertical and torsional position components. $B$ : back view of horizontal and vertical components. Displacement model fails to acquire target (or correct torsion) when retina is rotated torsionally out of its usual register with world. 

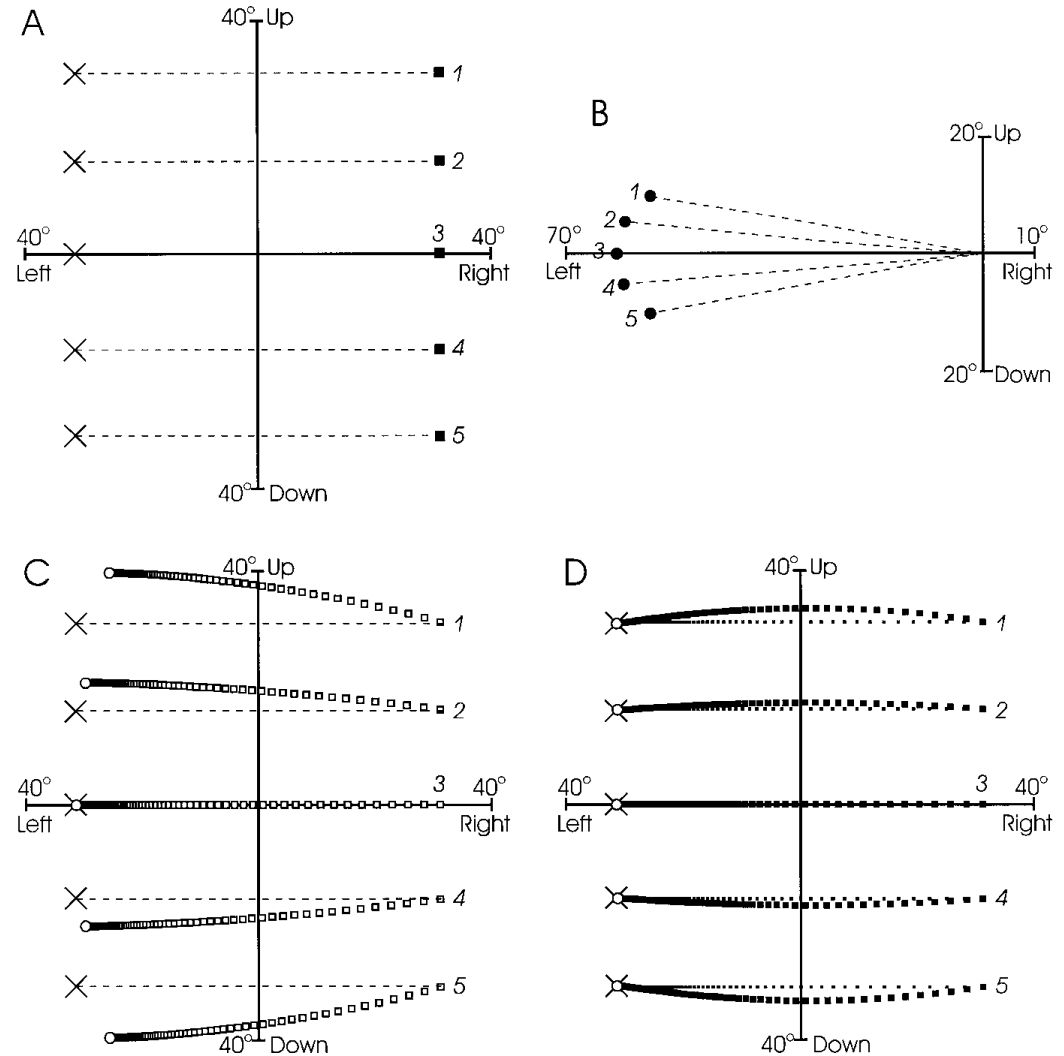

FIG. 10. Accuracy of 3-D displacement model and spatial model in oculomotor range for central 5 horizontal saccades simulated in Fig. 8. Horizontal and vertical components of gaze vectors are plotted relative to primary gaze direction and projected onto plane parallel to Listing's plane. $A$ : initial gaze and target directions. Gaze direction ( $\mathbf{a})$ was initialized at $30^{\circ}$ to right and at 5 different vertical levels (1-5) from $30^{\circ}$ up to $30^{\circ}$ down through $15^{\circ}$ intervals. Five simulated targets $(\times)$ were "presented" to left (in craniotopic coordinates) in opposite quadrant. $B$ : REs $(\bullet)$ computed from the 5 horizontal pairs of initial 3-D eye positions and target directions in $A$. $C$ : simulated gaze shifts ( $\square$ ) produced by displacement model. $D$ : simulated gaze trajectories produced by craniotopic plant version (large $\mathbf{a}$ ) and linear plant version ( small $\boldsymbol{\square}$ ) of spatial model. $\bigcirc$, final gaze directions. normal registry with the world (Fig. 9A), a purely upward target (in craniotopic coordinates) stimulated an oblique RE. In mapping this directly onto an equivalently tilted $\mathrm{ME}$, the displacement model thus generated an inaccurate saccade. In contrast, the 3-D reference frame transformation of the spatial model correctly compensated for this retinal torsion and produced an accurate saccade. Still, this effect would only be of passing interest to many investigators, so long as it only occurred with torsional eye positions and exaggerated positions beyond the oculomotor range (Fig. 6).

To test the reference frame problem in a more standard range, we simulated saccades to visual targets from a variety of positions in Listing's plane within $\mp 50^{\circ}$ of primary position. Figure 10 shows the basic result in the form of an experiment that could be performed in many oculomotor laboratories. This figure shows a simulated pattern of stimulus lights that has been used in many experiments: horizontally symmetric pairs of lights at several vertical levels, graphically represented by the tip of a unit vector pointing out from the origin and projected onto Listing's plane from behind. The origin thus indicates the primary gaze direction. In the simulated task, eye position was initialized in Listing's plane (as in Fig. 8) with gaze looking at the rightward targets ( $\square$, labeled 1-5), and the "subject", would be asked to saccade toward the leftward partners $(X)$. Normally, one would assume that these leftward lights would stimulate horizontal RE from each initial position (1-5), but this proved to be incorrect. Figure $10 \mathrm{~B}$ shows the same five leftward target directions converted into oculocentric coordinates by rotating them by the inverse of initial 3-D eye position at the five corresponding rightward targets. As illustrated, the horizontally displaced lights (in head/space coordinates) above and below primary gaze were displaced obliquely in oculocentric coordinates (O), fanning out from the horizontal in proportion to initial vertical position (this is the converse of the effect illustrated graphically in Fig. 3, where RE was held constant and objective target displacements were allowed to vary). Thus this task actually requires the subject to generate horizontal gaze shifts from nonhorizontal visual inputs.

Figure $10 C$ shows the performance of the 3-D displacement model at this task. The 2-D projections of unit gaze vectors are shown because these are very similar to the raw 2-D scleral search coil signals recorded in most laboratories (for 3-D data refer to Fig. 8A). Because this model mapped RE directly onto craniotopic position shifts, the resulting gaze trajectories $(\square)$ closely reflected the retinal inputs shown in Fig. 10B. As a result, the final gaze directions $(\bigcirc)$ missed the targets $(X)$ as a function of initial eye position. In the illustrated case, displacement-generated saccades from positions 2 and 4 missed the target by $4.5^{\circ}$, whereas 1 and 5 miss by $9.7^{\circ}$, mainly because of errors in direction. To generalize, the displacement model predicted that saccades would fan inappropriately outward (from primary position) in proportion to the component of eye position orthogonal to target displacement. (For example, the same centrifugal pattern was observed in simulations with vertically displaced targets at various horizontal eye positions.)

Figure $10 \mathrm{D}$ shows simulated gaze trajectories produced by both the 3-D spatial model, including both the craniotopic plant version (large $\mathbf{\square}$ ) and linear-plant version ( small $\mathbf{\square}$ ). Both versions generated gaze shifts that accurately acquired the targets $(X)$. The only difference between these two versions was that the linear plant version gave straight gaze 

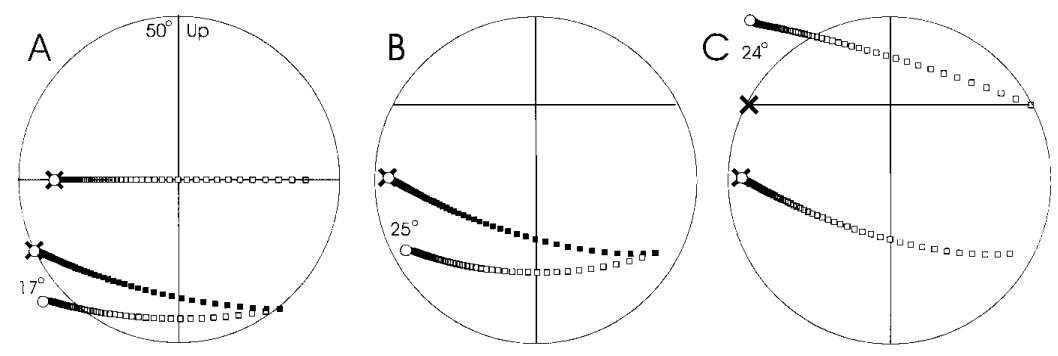

FIG. 11. Extreme examples of reference frame problem for visually guided saccades near edge of oculomotor range. Gaze vectors relative to primary gaze direction (intersection of horizontal and vertical axes) are projected onto plane parallel to Listing's plane as in previous figures. Outer circles: $\pm 50^{\circ}$ mechanical range; $\times$, desired target directions; $\bigcirc$, final gaze directions of simulated saccades; $\square$, simulated data generated by 3-D displacement model. Angle of final gaze error is indicated directly on figure; $\mathbf{m}$, simulated data generated by 3-D spatial model. $A$ : responses to $75^{\circ}$ leftward RE where primary position is at center of mechanical range. $B$ : response to $85^{\circ}$ leftward RE in case where primary position is elevated in mechanical range. $C$ : responses to $85^{\circ}$ leftward RE in displacement model that maps this onto oblique leftward-upward $\Delta E$ appropriate for bottom trajectory.

trajectories independent of gaze eccentricity, whereas the craniotopic plant (with internal implementation of saccade axis tilts for Listing's law) gave trajectories that curved as a function of gaze eccentricity. Thus the reference frame transformation in this model was both adequate and necessary to compensate for the nontrivial relationship between $\mathrm{RE}$, eye position, and objective target displacement independent of the detailed downstream neuromuscular implementation of Listing's law.

To quantify the maximum magnitude of gaze direction errors that could occur with a fixed visuomotor mapping, we simulated large saccades at the edge of the oculomotor range. This is shown in Fig. 11 with the use of the same plotting conventions as in Fig. 10. Gaze errors produced by the displacement model $(\square)$ are indicated in the figure. Again, the maximum error will depend on eccentricity from primary position and the magnitude of RE, which in turn depend on the oculomotor range and the maximum range of peripheral vision, which approaches $90^{\circ}$ for temporally displaced targets, depending on the subject. Figure $11 \mathrm{~A}$ shows the effect of the displacement model mapping $75^{\circ}$ leftward RE onto $75^{\circ}$ leftward change in eye position both from a purely rightward position and from a position at the lower right edge of the $\mp 50^{\circ}$ range. The latter saccade missed the target by $17^{\circ}$, although presumably in real life the error would be greater if this saccade jammed at the edge of the mechanical range. An accurate movement generated by the 3-D spatial model ( $\mathbf{a})$ is also provided for comparison.

This is an extreme case if we assume that the primary position defined by Listing's law is at the center of the oculomotor range [note that the reference-frame effect imposed by the half-angle rule of Listing's law (Fig. 4) is measured relative to primary position]. However, experimental measurements suggest that the primary position is widely distributed, sometimes even near the periphery, through the oculomotor ranges of different human subjects (e.g., Tweed and Vilis 1990a) and is usually near the top center of the primate oculomotor range (e.g., Crawford 1994; Hepp et al. 1993). Figure $11 B$ indicates the latter situation, in which the primary position (at the intersection of the axes) is located three-quarters of the way up in the mechanical range (outer circle). In this subject the saccades simulated in the lower range of Fig. 10 (where the displacement model produced errors of $5-12^{\circ}$ ) would occur near the cen- ter of the range. Furthermore, as illustrated by the simulated response (Fig. $11 B$ ) to an $85^{\circ}$ leftward RE (probably close to the physiological maximum) from a downward-rightward position, the displacement model predicts gaze errors $>25^{\circ}$. Could the displacement model not learn to overcome this effect? Figure $11 C$ illustrates the hypothetical case in which the displacement model has "learned" to map a horizontal RE onto an oblique change in eye position (bottom trajectory) to produce an accurate saccade ( simulated by adding bias terms onto ME). However, because the displacement model requires this to be a fixed mapping irrespective of initial eye position, the model now fails to generate accurate movements in response to horizontal RE at the vertical level of primary position (top trajectory).

Table 1 quantitatively summarizes the possible range of gaze errors produced by the 3-D displacement model, compared with the spatial model, through a wide range of positions and REs in $10^{\circ}$ steps. To reduce the complexity of this table, we have only included initial eye positions in Listing's plane, with $10^{\circ}$ steps in horizontal RE and vertical eye position, with saccades centered across the midline. (The same numbers will hold for any direction of RE with position varying along the orthogonal direction.) From these tabulated data it is evident that the spatial model only produced very small errors, even for large REs and gaze eccentricities (these consisted of tiny undershoots that arose from the algorithm that terminated saccades). The tabulated data also indicate that the displacement model functioned reasonably well for saccades within the central range within Listing's plane. However, as both RE and gaze eccentricity increased, the performance of the displacement model diverged rapidly from the spatial model, particularly when both were large. As illustrated in the preceding text, the inaccuracy of the displacement model was mainly due to systematic errors in saccade direction.

\section{Invasive tests of the visuomotor interface}

Saccadelike displacements of eye position have been evoked by electrical stimulation of numerous "visual" and "motor" sites in the brain. On the basis of these movements alone, it was difficult to know whether such stimulation has defined a visual target (i.e., a phosphene) in retinal coordinates or a command that is uniquely motor in charac- 
TABLE 1. Gaze direction errors produced by the displacement vs. spatial model for various retinal errors and gaze eccentricities

\begin{tabular}{|c|c|c|c|c|c|c|c|}
\hline & $0^{\circ} \mathrm{P}$ & $10^{\circ} \mathrm{P}$ & $20^{\circ} \mathrm{P}$ & $30^{\circ} \mathrm{P}$ & $40^{\circ} \mathrm{P}$ & $50^{\circ} \mathrm{P}$ & $60^{\circ} \mathrm{P}$ \\
\hline $20^{\circ} \mathrm{RE}$ & $0.03(0.02)$ & $0.31(0.02)$ & $0.71(0.03)$ & $1.24(0.03)$ & $1.93(0.03)$ & $2.78(0.04)$ & $3.78(0.05)$ \\
\hline $40^{\circ} \mathrm{RE}$ & $0.05(0.06)$ & $1.19(0.07)$ & $2.5(0.08)$ & $3.86(0.11)$ & $5.44(0.14)$ & $7.24(0.18)$ & $9.25(0.24)$ \\
\hline $50^{\circ} \mathrm{RE}$ & $0.07(0.17)$ & $1.82(0.18)$ & $3.71(0.21)$ & $5.71(0.24)$ & $7.88(0.28)$ & $10.24(0.35)$ & $12.81(0.43)$ \\
\hline $60^{\circ} \mathrm{RE}$ & $0.08(0.36)$ & $2.56(0.38)$ & $5.18(0.39)$ & $7.88(0.45)$ & $10.73(0.49)$ & $13.74(0.58)$ & $16.91(0.69)$ \\
\hline $70^{\circ} \mathrm{RE}$ & $0.09(0.65)$ & $3.40(0.64)$ & $6.84(0.68)$ & $10.34(0.73)$ & $13.94(0.80)$ & $17.66(0.90)$ & $21.50(1.04)$ \\
\hline
\end{tabular}

Values are gaze direction errors (in deg) for displacement model. Values in parentheses are for spatial model. Only $10^{\circ}$ steps in retinal error (RE) and position (P) are shown, where RE is along 1 cardinal direction (e.g., horizontal) and position is along the orthogonal direction (e.g., vertical). To further narrow the possible combinations and keep the saccades centered in the oculomotor range, initial horizontal position was always set at $-1 / 2$ the angle of RE. Gaze error was computed by taking the dot product between actual and desired final gaze directions.

ter. To provide a formal geometric test between what is uniquely visual and what is uniquely motor, we simulated the predicted effects of stimulating a fixed RE versus a fixed $\mathrm{ME}$ in both models ( single-unit recording will be considered in the DISCUSSION ). Figure 12 shows simulated gaze trajectories (top) and 3-D position vectors (bottom) where eye position was initialized at various points within Listing's plane.

First, we consider the predictions of our 3-D spatial model, which will hold if real saccades are accurate and obey Listing's law for arbitrary eye positions and REs (Figs. 7-11). In Fig. $12 \mathrm{~A}$, gaze direction was always initialized at $30^{\circ}$ to the right, but at seven different vertical levels (1-7). The illustrated simulations show the expected result of stimulating a fixed $\mathrm{ME}$ command ( $\Delta E_{\mathrm{i}}$, downstream from the position-dependent visuomotor transformations in Fig. $5 B$ ) that specifies a $60^{\circ}$ leftward change in eye position. This translated into a near-constant displacement in gaze direction relative to the head (Fig. 12A) independent of initial eye position, such that the gaze trajectories stayed in parallel register.
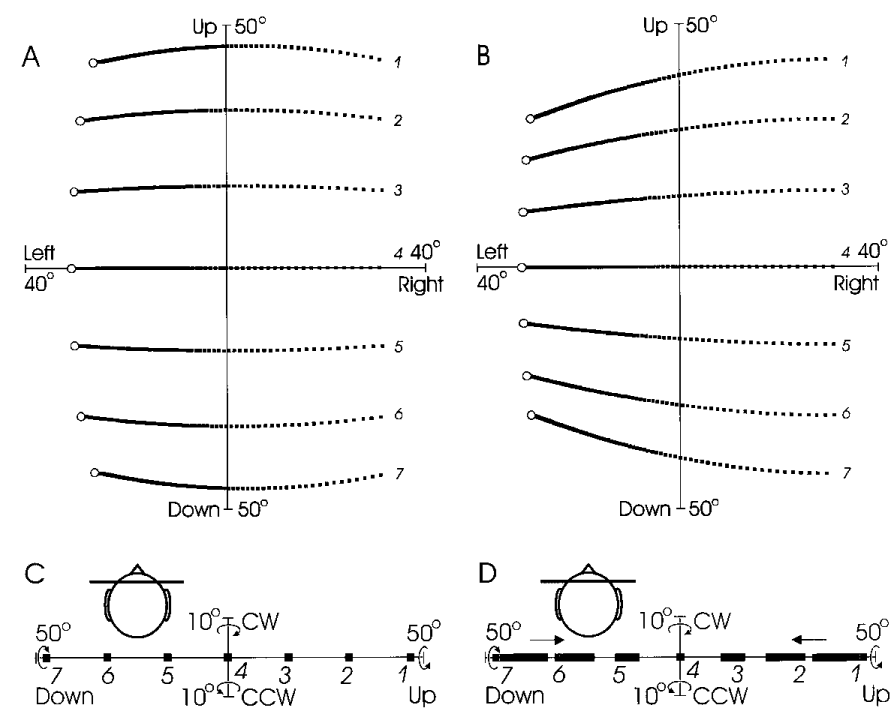

FIG. 12. Simulated gaze shifts produced by stimulating hypothetic neural sites that encode $(A) 60^{\circ}$ leftward change in eye position in Listing's plane and $(B) 60^{\circ}$ leftward RE. Gaze direction is plotted in Listing's coordinates. In each case, eye position was initialized in Listing's plane with gaze direction $30^{\circ}$ to right at 7 vertical levels (1-7) from $45^{\circ}$ up to $45^{\circ}$ down. $A$ could be simulated by either spatial model or displacement model, but $B$ could only be simulated by spatial model.
In contrast, stimulation of a fixed RE produced a characteristic position-dependent pattern of eye movements. Figure $12 B$ shows simulated activation of $60^{\circ}$ leftward RE (upstream from the reference frame transformation) from the same seven initial positions as in Fig. 12A. In this case, the downstream reference frame transformation computed a different ME command, corresponding to the same RE, for each initial eye position. As a result, the simulated gaze displacements showed a centripetal or converging pattern. ${ }^{3}$ The greater the magnitude of $\mathrm{RE}$, the greater was this converging effect (Fig. 3), until at $90^{\circ}$ horizontal RE the eye would converge on a single position from all initial secondary positions in the vertical range (Fig. 7, $A$ and $C$ ). Thus the spatial model predicts that stimulation of RE and ME will produce two distinct movement patterns. The centrifugal position-dependent pattern in Fig. 12B would be consistent with a true visual code upstream from an eye-to-head reference frame transformation, whereas the position-independent pattern illustrated in Fig. 12A would indicates a downstream motor code.

The predictions of the displacement model were less instructive (these predictions would hold only if the system shows the behavioral errors predicted by this model in Figs. 6 and $8-11$ ). Stimulating $60^{\circ}$ leftward ME gave the same fixed-vector result as in Fig. 12A. (This should not be surprising, because both models share similar operations downstream from the ME command.) However, stimulation of $60^{\circ}$ leftward RE also gave the same fixed-vector result, again indistinguishable from that in Fig. 12A. This occurred because the displacement model trivially mapped $60^{\circ}$ leftward $\mathrm{RE}$ onto a $60^{\circ}$ leftward change in eye position independent of initial eye position. Thus the displacement model predicted that stimulus-evoked "saccades" will always show a fixed-vector position-independent pattern and offered no means to test between visual and motor codes.

One would also like to know the location of the neural 2-D-to-3-D transformation, i.e., the Listing's law operator

\footnotetext{
${ }^{3}$ Note that, as in our behavioral simulations, these effects are centered symmetrically about the primary gaze direction defined by Listing's law. This occurs in our model because RE is transformed directly into a set of motor codes in Listing's coordinates and would change if we placed any additional coordinate transformation between these levels. Thus this is also subject to experimental verification. The pattern of symmetry in Fig. 12B would indicate that Listing's primary position is the reference position for the saccade generator, where oculocentric and craniotopic coordinates come into alignment.
} 

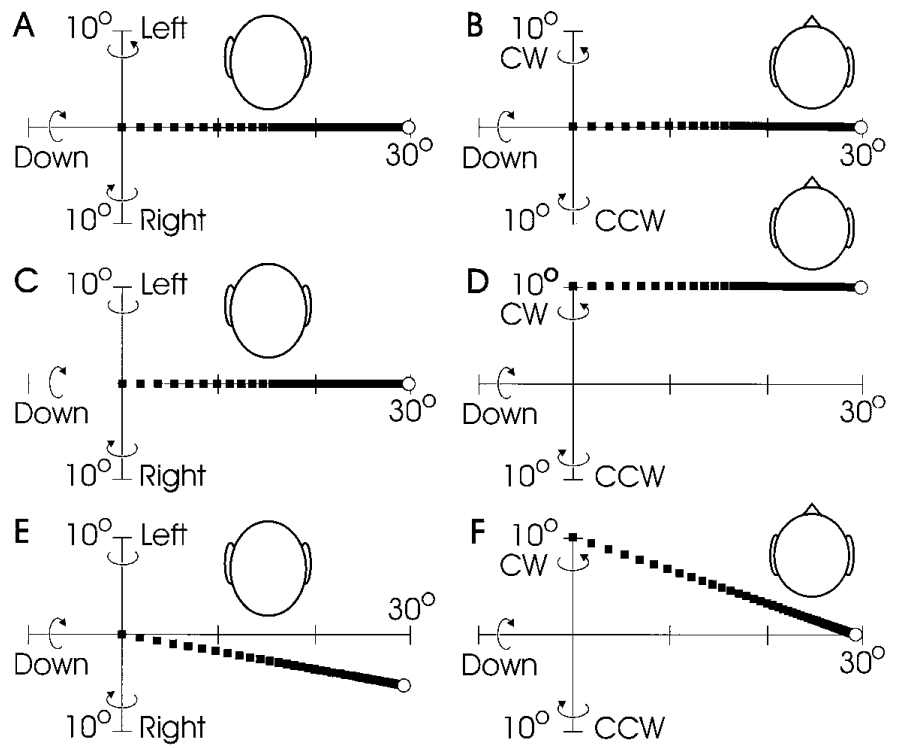

FIG. 13. Simulated eye position trajectories produced by brain stimulation in presence of initial torsion. 3-D eye position vectors are plotted in Listing's coordinates as viewed from behind Listing's plane (left) and above Listing's plane (right). $A$ and $B$ : with eye position initialized at primary position, movements evoked by $30^{\circ}$ upward RE and $30^{\circ}$ change in eye position are indistinguishable. $C$ and $D$ : evoked $30^{\circ}$ upward change in eye position with eye position initialized at $10^{\circ}$ clockwise torsion. $E$ and $F$ : evoked $30^{\circ}$ upward RE ( simulated in spatial model upstream from spatial transformations ) with eye position initialized at $10^{\circ}$ clockwise.

(Eq. 15). In the model of Tweed and Vilis (1990b), this operation was immediately followed by a multiplicative computation of a fixed-axis ME command, perhaps coded by the superior colliculus. Tweed and Vilis thus predicted that stimulation of the superior colliculus would produce position-dependent violations of Listing's law, which was contradicted by the results of Van Opstal et al. (1991). On the basis of these results it has been widely assumed that Listing's law is entirely implemented downstream from the superior colliculus. However, our spatial model makes an entirely different set of predictions. Figure $12, C$ and $D$, shows 3-D eye position vectors for the gaze trajectories discussed in the preceding text, as viewed looking down Listing's plane from above the head. Note that, unlike the Tweed-Vilis model, even stimulation of a fixed ME downstream from the Listing's law operator (Fig. 12C) produced trajectories that stayed in Listing's plane, independent of initial eye position. Thus, if the oculomotor system functions like our model, such a result (Van Opstal et al. 1991) would be ambiguous with respect to the 2-D-to-3-D transformation (Eq. 15).

Figure 13 provides the unambiguous test necessary to determine whether displacement codes are upstream or downstream from the Listing's law operator (in our model). This figure shows simulated 3-D eye position trajectories generated by the spatial model as viewed from behind the head (left) and above the head (right). Figure 13, $A$ and $B$, shows a simulated $30^{\circ}$ upward saccade starting from primary position. Suppose that this was evoked by stimulation of some site in the brain. From this data, it would again be impossible to know whether this site encoded $30^{\circ}$ upward RE (Fig. $5 B$ ) or $30^{\circ}$ upward $\mathrm{ME}\left(\Delta E_{\mathrm{i}}\right.$, Fig. $5 B$ ), but this was no longer true in the presence of torsion (Fig. 13, $C-F$ ). Let us first suppose that this hypothetical site encodes $\mathrm{ME}$, as simulated by activating a zero-torsion $30^{\circ}$ upward $\Delta E_{\mathrm{i}}$ downstream from the spatial transformations. Starting from a $10^{\circ}$ clockwise position (Fig. 13, $C$ and $D$ ), this produces the same fixed-vector position trajectory as before (compare Fig. 13, $A$ and $C$ ), displacing eye position parallel to Listing's plane without correcting the initial torsion (Fig. 13D).

In contrast, if this hypothetical site encodes RE ( simulated by inputting $30^{\circ}$ upward RE upstream from the spatial transformations ), then this initial $10^{\circ}$ clockwise torsion should be compensated for in two distinct ways (Fig. 13, $E$ and $F$ ). First, from the behind perspective (Fig. 13E) the resulting trajectory is rotated $10^{\circ}$ clockwise, reflecting the correct downstream reference frame transformation from upward (oculocentric) RE to oblique (craniotopic) ME. Second (with the head upright), the resulting position trajectory should now possess a torsional component (driven by a finite torsional component in $\mathrm{ME}$ ) that corrects the initial torsional deviation from Listing's plane (Fig. $13 F$ ), reflecting the downstream comparison between initial 3-D eye position and desired position in Listing's plane.

\section{IS C US SION}

\section{Implications for behavior and modeling}

Contrary to a previous hypothesis (Crawford and Vilis 1991), the visuomotor lookup table of the displacementfeedback model does not preclude correct computation of the torsional velocity axis tilts required to keep eye position in Listing's plane (if, as in Fig. $8 B$, ME codes 0 torsion $\Delta E$ and the position-dependent half-angle rule for these axes is implemented downstream). However, a fixed visuomotor mapping does preclude the correct solution of two other important physiological problems. First, the reference frame problem: in implementing the half-angle rule for Listing's law, we prevent saccades from operating in the same oculocentric reference frame as RE. This precludes a trivial physiological solution to the 3-D eye-position-dependent relationship between objective target displacement and RE (Figs. 3 and 4). A direct mapping of RE onto a kinematically correct eye position displacement in Listing's plane would thus result in centrifugal (outward) errors in saccade direction independent of plant characteristics. This could potentially produce maximum gaze errors of $17-25^{\circ}$, depending on the magnitude of RE and the orthogonal components of eye position relative to primary position (Figs. 6 and $8-11$, Table 1). Second, the trivial 2-D-to-3-D transformation imposed by a visuomotor lookup table fails to deal correctly with the degrees of freedom problem, particularly when eye position deviated torsionally from Listing's plane (Fig. 9). Thus we conclude that a visuomotor lookup table could only provide correct saccades in very restricted circumstances, i.e., with the head fixed and eye position confined to the central range of Listing's plane.

To make the displacement-feedback model work in 3-D, we had to replace its visuomotor lookup table with a set of nontrivial position-dependent transformations. As we have seen, the central reason why these internal "spatial', transformations are required is that, contrary to the common wis- 
dom in 1-D and 2-D studies, visual and motor displacement spaces have fundamentally different geometric properties. RE is defined by its input geometry (Fig. 2) to be 2-D and oculocentric, whereas ME is defined by its output geometry be 3-D and craniotopic. Rather than abandon the many advantages of the original displacement-feedback model, we have solved the resulting computational problems by incorporating a multiplicative, position-dependent reference frame transformation and nontrivial 2-D-to-3-D transformation between RE and ME (Fig. 5B). The resulting "spatial-displacement hybrid' ' model utilized the distinctive double comparison with eye position feedback first used in the 1-D spatial model (Fig. 1), but in 3-D these operations are nonredundant and indispensable for accurate and kinematically correct saccades (Figs. 7-11).

Do real saccades show this ideal behavior, even at their mechanical limits? This can only be answered by experimental data. Correction of torsional deviations from Listing's plane by subsequent saccades has already been well described (e.g., Crawford and Vilis 1991; Van Opstal et al. 1996), so it is fairly clear that the system uses a correct 2-Dto-3-D transformation, but data relevant to the visuomotor reference frame problem are less available. No one has ever reported a pattern of errors like that predicted by our 3-D displacement model, but neither has anyone set out to look for such a pattern. Studies of saccades directly toward visual targets have reported slight undershoots (Yarbus 1967) and overshoots (Fuchs 1967) but not direction errors. The only comparable effect that we have located in the literature was observed in saccades to remembered targets (Gnadt et al. 1991; Stanford and Sparks 1994; White et al. 1994), but the pattern of errors was essentially centripetal (inward), opposite to the pattern of errors predicted by the displacement model. Thus a reexamination of saccade accuracy from all initial eye positions seems to be warranted, as we have outlined with Fig. 10. Although it seems unlikely that the real system would produce errors as large as those predicted by the displacement model, it is possible that some degree of under- or overcompensation for eye position might occur in the absence of visual feedback. If so, it would be of interest to see whether the system uses secondary saccades to correct for these errors or if it would be completely "unaware' of such mistakes.

Assuming that saccades are reasonably accurate and that Listing's law is obeyed from all initial eye positions, then the basic geometry of the oculomotor system would require us to reject the visuomotor lookup table hypothesis. It may remain useful to model the majority of small to medium retinal saccades ( say $\leq 25^{\circ}$ ) in Listing's plane with a simple displacement-feedback model (or the equivalent) (e.g., Scudder 1988), but at best this model would only be indistinguishable from the correct model within this limited range (to think otherwise is to propose 2 separate saccade generators for small and large REs). Moreover, even small REs must be compared with 3-D eye position when Listing's law is violated (Fig. 9). Because this occurs continuously in the head-free conditions in which we have evolved (Crawford and Vilis 1991; Tweed et al. 1995), it seems unlikely that this comparator would suddenly disengage when the head is fixed to an experimental bite bar.

\section{Implications for visuomotor neurophysiology}

Supposing that the oculomotor system does show the ideal behavior predicted, where then are the position-dependent neural implementations of the requisite spatial transformations? Because the retina definitely encodes 2-D oculocentric variables, and the reticular formation burst neurons almost certainly use a 3-D craniotopic coordinate system (Crawford and Vilis 1992; Henn et al. 1989), the reference frame transformation and 2-D-to-3-D transformation have to occur somewhere between these two points. The explicit 3-D eye position information needed for this seems to exists only in the brain stem reticular formation (e.g., Crawford 1994). However, efference copies of eye position also exist in the form of implicit gain fields in higher visuomotor structures such as posterior parietal cortex (e.g., Andersen et al. 1985) and superior colliculus (Van Opstal et al. 1995). Furthermore, it has been demonstrated that such gain fields could, at least in theory, effect an eye-to-head reference frame transformation similar to that in our model (Van Opstal and Hepp 1995; Zipser and Andersen 1988).

The distributed gain field model (e.g., Zipser and Andersen 1988) has sometimes been criticized because it still specifies explicit target direction in its output, whereas physiological saccade commands seem to overwhelmingly specify displacements (e.g., Goldberg and Bruce 1990; Moschovakis and Highstein 1994; Robinson 1972; Schiller and Stryker 1972; Sparks 1988; Waitzman et al. 1991). However, it is not hard to envision a neural network version of our spatial model in which 2-D oculocentric RE and 3-D eye position are the inputs and 3-D craniotopic ME is the output, without ever explicitly computing desired gaze direction in head coordinates (e.g., see Van Opstal and Hepp 1995). In contrast to the 2-D version, this model would require displacement signals to receive inputs from the orthogonal components of position to compute the correct relation between $\mathrm{RE}$ and $\mathrm{ME}$ (Figs. 9-11). For example, even 2-D displacement codes would require torsional eye position gain fields to handle the situation portrayed in Fig. 9. However, even if one identified a cortical or subcortical site where neurons showed these properties, it would still be very difficult to directly prove the function of such implicit position dependencies.

To this end, we have provided, by stimulating the more explicit and experimentally accessible displacement codes, a set of experimental predictions (Figs. 12 and 13) that could provide such a proof. By determining the position-dependent geometry of the gaze displacements produced by stimulating sites in the superior colliculus, brain stem, visuomotor cortex, and cerebellum, it should be possible to determine their placement relative to the spatial transformations proposed in our model (Fig. $5 B$ ). In particular, stimulation of a pure RE signal (upstream from the reference frame transformation) should produce a position-dependent, centripetal (pseudo-goal-directed) pattern of gaze shifts, whereas stimulation of ME (downstream from the spatial transformations) should produce parallel, position-independent gaze shifts (Fig. 12). This is reminiscent of the debate between investigators who have found that stimulation of various neural sites produced fixed-vector saccades as opposed to a pseudogoal-directed centripetal pattern of saccades (e.g., Freedman et al. 1996; Robinson 1972; Schiller and Stryker 1972). 
The position-dependent patterns of RE and ME (Figs. 12 and 13) also provide predictions for single-unit recording. This should be further specified as two separate types of coding: the visual tuning of a cell (i.e., its visual receptive field) and its motor tuning (i.e., correlation of its activity to an optimal saccade vector, which should be closely related to the stimulation-evoked vector). For example, tectoreticulospinal neurons in the intermediate and deep layers of the superior colliculus are involved in gaze shifts but also have visual receptive fields (e.g., Munoz et al. 1991). Because visual tuning is determined by upstream transformations and motor tuning is determined by downstream transformations, these need not be equal.

The 3-D displacement model predicts that visual and motor tuning indeed will diverge. In any model, one would expect early visual neurons to be tuned to raw RE, which would require their visual tuning direction (in craniotopic coordinates) to vary systematically with eye position (as in Figs. $12 B$ and $13 E$ ). Because this information would be passed downstream without position-dependent processing (in a fixed visuomotor mapping), motor cells like tectoreticulospinal neurons would show the same position-dependent pattern of visual tuning. By corollary, visual and motor cells would also share the same motor tuning, but this would now be the fixed-vector pattern determined by the downstream motor transformations (Figs. $12 A$ and 13, $A$ and $C$ ). Thus visual and motor tuning would diverge as a function of eye position within individual neurons at all levels, in the same way that the trajectories in Fig. $12, A$ and $B$, diverge. For example, a tectoreticulospinal neuron might code leftward gaze shifts from all positions but be visually tuned to centripetally tilted oblique directions when looking up or down. This seems to be an odd prediction, but it is the necessary physiological correlate of the visuomotor inaccuracy predicted by the 3-D displacement model (Figs. 6, 10, and 11).

The predictions of the 3-D spatial model are quite different. Because of the progressive position-dependent processing in this model, it predicts a change in both visual and motor tuning in the transformation from RE to ME while maintaining registry between these codes at all levels. For example, the directional tuning (both visual and motor) of visual units would show the position-dependent modulations predicted for geometric RE (Figs. $12 B$ and 13, $A$ and $E$ ). In contrast, the position-dependent transformations downstream would ensure that both the visual and motor tuning of units coding ME would show the fixed-vector pattern (Fig. 12A). Thus this model provides a geometric definition for what is visual and what is motor in the oculomotor system, and its reference frame transformation ensures a correct registry between sensory and motor tuning of cells at both levels.

To illustrate the potential impact of this 3-D spatial theory on our understanding of visuomotor physiology, we briefly consider two possible results of performing our cell recording and stimulation tests (Figs. 12 and 13) on the superior colliculus. (These are just 2 extreme possibilities with fairly clear didactic value; it is equally possible that these transformations occur in a gradual progression through several stages of processing or within the colliculus itself.) One possibility is that even the deep layers of the colliculus encode geometric RE. This would suggest that both the 2-D-
to-3-D and the reference frame transformations somehow occur in the one or two synapses between the latter and the reticular formation burst neurons (if so, then the ME might only exist at the abstract level of the synaptic inputs to burst neurons). This seems to be consistent with recent data suggesting that the superior colliculus encodes a visual signal independent of detailed saccade metrics (Stanford and Sparks 1994) and with the view that upstream structures maintain target location in a purely oculocentric frame (Duhamel et al. 1992). Furthermore, this view is easy to reconcile with the idea that the colliculus encodes a gaze command for both the eye and the head (Freedman et al. 1996; Munoz et al. 1991). In general, this hypothesis represents the "parallel processing view" in which fairly unprocessed sensory geometries are used down to a relatively late stage and then processed separately for each motor system.

Another possibility is that RE is only encoded in strictly visual structures such as the superficial layers of the superior colliculus, whereas the deep motor map of the colliculus encodes $\Delta E$. Because torsional components of rapid eye movements are not affected by inactivation of the colliculus (Hepp et al. 1993; Van Opstal et al. 1991), this option would require that the torsional component of $\Delta E$ be implemented in a parallel quick-phase generator. This hypothesis is intriguing because it would require a position-dependent remapping between the sensory and motor maps of the colliculus for every saccade. If correct, it would account for part of the complexity of the mainly indirect connections between the superficial and deep colliculus via cortical visuomotor structures (Moschovakis and Highstein 1994; Sparks 1988; Wurtz and Albano 1980). It is thus of interest that one of these intermediate structures, the posterior parietal cortex, has already been implicated in an eye-to-head reference frame transformation (Andersen et al. 1985). In general, this scheme would allow the eye-to-head reference frame transformation to occur at an early processing stage for all motor systems (Flanders et al. 1992) and even perception (Haustein and Mittelstaedt 1990; Howard 1982) in a single global stage.

This "global processing" view seems to be consistent with the suggestion that visual target locations are remembered in craniotopic coordinates by comparing visual signals and eye position signals upstream from the motor layers of the colliculus (Andersen et al. 1985; Schlag et al. 1989; Sparks and Mays 1983). Similarly, the former parallel processing view seems to be more consistent with alternative theory that target direction is remembered as retinotopic representations that shift as a function of saccade metrics (Duhamel et al. 1992; Goldberg and Bruce 1990). However, the position-dependent transformations in our spatial model may have nothing to do with spatial memory and can potentially be combined with either of the preceding models without contradiction. Our work simply states that a $3-D$ positiondependent eye-to-head reference frame transformation and 2-D-to-3-D transformation are necessary for saccade execution.

\section{Listing's law}

Despite a decade of intensive research, considerable confusion still surrounds the issues related to Listing's law. 
This seems to have arisen from an implicit assumption that Listing's law is implemented in a single physiological stage. In fact, Listing's law is closely related to three different computational processes that could potentially be physiologically separate: the 2-D-to-3-D transformation (called the Listing's law operator by Tweed and Vilis 1990b), the implementation of the half-angle rule for torsional axis tilts (Tweed and Vilis 1990a), and the problem (imposed by the rules of rotational kinematics) of matching 3-D velocity and position signals (Tweed and Vilis 1987). For example, in the model of Tweed and Vilis (1990b), the Listing's law operator and half-angle rule were implemented in close succession, but in our model (Fig. 5B) the half-angle rule is implemented within the neuromuscular mechanisms for 3 -D velocity-position matching, several steps downstream from the Listing's law operator. These changes clarify that colliculus stimulation results (Hepp et al. 1993; Van Opstal et al. 1991) show that the half-angle rule is implemented downstream but cannot yet comment on the location of the 2-D-to-3-D transformation (Fig. 12).

A similar problem has crept into the debate over muscular versus neural implementation Listing's law (Crawford and Vilis 1991; Demer et al. 1995; Schnabolk and Raphan 1994; Tweed et al. 1994). Despite the misgivings of some authors (e.g., Demer et al. 1995), the 3-D behavioral data demonstrates quite definitively that the muscles do not confine the eye to Listing's plane and thus cannot solve the degrees of freedom problem (Crawford and Vilis 1991, 1995). The real issue here is whether or not the plant itself, through some muscular (Fig. 4) or passive ${ }^{2}$ mechanism, implements the half-angle rule and perhaps even the solves the velocityposition problem for saccades. To be fair to both views, we included both a linear plant (that solves both of these problems) and a conceptually less sophisticated plant (that required neural solutions) into our models. Both versions maintained eye position in Listing's plane and generating the right velocity axis tilts (Fig. 8). However, these 3-Dto-3-D transformations only facilitated Listing's law if both the initial eye position vector and the ME vector were parallel to Listing's plane. They could provide no means to prevent torsional brain stem signals from violating Listing's law or to correct such violations in the absence of a torsional neural signal (Fig. 9).

What then, does Listing's law say about neural control signals? If initial eye position were always confined to Listing's plane, then, as our displacement model demonstrates (Fig. 8), Listing's law would result "naturally" (Clement 1991; Schnabolk and Raphan 1994), i.e., from a trivial visuomotor mapping where burst neurons encode 2-D movement vectors in Listing's coordinates (Crawford and Vilis 1992; Robinson and Zee 1981). However, such schemes miss the main point: there is nothing trivial about aligning burst neuron vectors with Listing's plane. Burst neurons utilize a complete 3-D coordinate system (Crawford and Vilis 1992; Henn et al. 1989) that frequently must encode systematic torsional components when the head is free to move (Crawford and Vilis 1991; Henn et al. 1989; Tweed et al. 1995). Thus the key question is how the brain chooses the correct 3-D pattern of burst neuron activity (Crawford and Vilis 1995; Hepp 1994; Tweed and Vilis 1987).

In the current paper we argue that burst neurons are acti- vated by an ME signal that is derived (either explicitly or in some distributed fashion) from a comparison between current 3-D eye position and the output of a Listing's law operator (Tweed and Vilis 1990b). This Listing's law operator is the definitive 2-D-to-3-D transformation in our model. Van Opstal et al. (1996) recently employed a similar conceptual scheme to explain how the brain stem and cerebellum might generate a torsional error signal to correct minute head-fixed deviations from Listing's plane. Consistent with our model, this mechanism appeared to be independent of the implementation of the half-angle rule torsional velocity components (Van Opstal et al. 1996). However, our spatial model suggests that the test illustrated in Fig. 13 will be required to demonstrate the functional location of the Listing's law operator relative to the superior colliculus. Furthermore, our spatial model suggests that this operator does not simply add a torsional signal onto a 2-D visual signal or (despite its name) strictly concern Listing's law. In computing all three components of desired 3-D eye position from visual signals, this operator would participate in all aspects of gaze control, determining the complete width, shape, orientation, and extent of the eye-in-head position range. Listing's plane would simply reflect a default range, as we have modeled it, where gaze is directed toward distant targets and the head is upright and motionless.

\section{A P PENDIX}

\section{Mathematical implementation}

The algorithms illustrated in Fig. 5 were implemented with the use of programs written in MICROSOFT QUICKBASIC 4.5. The mathematical representations within these algorithms were largely constrained by the physiology discussed in the MODELS section. However, a number of unconstrained operations ( such as rotation of a vector) remained that could potentially be implemented with the use of the algebra of, e.g., rotation matrices, quaternions, and various classes of 3-D vectors (Funda and Paul 1990; Haslwanter 1995; Haustein 1989; Hepp et al. 1993; Tweed and Vilis 1987). Our specific goal was to model input-output relations at the behavioral level (Figs. 6-11) and at the level of major geometric transformations between neural populations and structures (Figs. 12 and 13 ). Therefore, in cases in which the choice of implementation did not affect experimental predictions at this level, our choice of math was largely guided by convenience, as detailed in the following text.

\section{Coordinate systems}

We used a head-fixed, orthogonal, right-handed coordinate system (in which the 1st, 2nd, and 3rd vector components are measured along the torsional, horizontal, and vertical axes) to implement our math and display data. For physiological realism (Crawford 1994; Crawford and Vilis 1992; Tweed et al. 1990; Van Opstal et al. 1991) we used a head-fixed coordinate system where the torsional axis was orthogonal to and the horizontal and vertical coordinate axes were aligned with Listing's plane. We ignored internal "matrix" coordinate transformations, because these have been dealt with at length elsewhere (e.g., Crawford 1994; Robinson 1982) and could easily be incorporated into the model without adding anything new. Our use of Listing's coordinates specified that 1 ) the "zero" eye position or gaze direction always referred to Listing's primary position, 2) a zero torsion vector was parallel to Listing's plane, and 3 ) the horizontal meridian of the retina was defined to be the arc of retina that intersects with the horizontal 
plane orthogonal to Listing's plane when the eye is at the primary position. The latter horizontal plane was further specified to align with the earth-horizontal when Listing's plane is aligned with the gravity vector. When the head was defined to be fixed in this orientation, our coordinates for target position relative to the head and relative to space became trivially interchangeable.

\section{Implementation of the displacement model}

Because the internal workings of the 3-D displacement model (Fig. 5A) consisted entirely of linear operations, eye position was represented with the use of simple 3-D angular position vectors in this model, i.e., with length directly proportionate to the angle of rotation relative to primary position. In contrast, several operations relating to actual target direction, eye position, and eye velocity were rotational and multiplicative. For these external operations we used quaternions, because their algebra applies easily to rotations and is freely available in the oculomotor literature (e.g., Tweed and Vilis 1987; Tweed et al. 1990; Westheimer 1957). In brief, quaternions are composed of a scalar part $\left(q_{0}\right)$ equal to the cosine of half the angle of rotation, and a vector part $(q)$, which is qualitatively similar to the angular position vectors described in the preceding text, but its length is equal to the sine of half the angle of rotation. With the use of this relationship, quaternions and angular position vectors were easily interconverted when required.

At the beginning of each simulation internal $(E)$ and actual $(\underline{E})$ eye position were initialized to an initial value and input to the model (underlining indicates external kinematic measures). When a target direction in craniotopic coordinates was used as the initial visual input, it was specified as a unit vector parallel to desired gaze direction in head-fixed Listing's coordinates $\left(G d_{\text {head }}\right)$. (The vertical and horizontal angles of target direction provided in the text refer to angles between the target direction vector and primary gaze as projected onto the horizontal and sagittal planes in the coordinates defined.) To compute $G d_{\text {eye }}$ (Fig. 2) and thus specify the site of retinal stimulation, $G d$ head was rotated by the inverse of current eye position with the use of quaternion conjugation

$$
\underline{G d}_{\text {eye }}=\underline{E}^{-1}\left(\underline{G d}_{\text {head }}\right) \underline{E}
$$

where the vector $(1,0,0)$ represents current gaze $\left(G_{\text {eye }}\right)$, or the incident line of foveal stimulation, and the subscript "eye" denotes a coordinate system based on the plane orthogonal to this vector. If one knew the precise shape of the retina and its distance from the optical node, one could compute the actual optically inverted retinal location of this stimulus from $G d_{\text {eye }}$, but because each retinal site will thus have a one-to-one mapping to $\underline{G d}$ eye, and because this map will be used as a lookup table for target locations, this computation would be redundant for modeling saccade control. RE can thus be interpreted as the 2-D projection of $\underline{G d}$ eye on the gazeorthogonal plane. To compute the RE between current and desired gaze, the cross product was used

$$
R E=\left(\underline{G}_{\text {eye }} \times \underline{G d}_{\text {eye }}\right)
$$

This gave RE, scaled as the sine of an angle. To put this into a degree scale, normalized RE was multiplied by the inverse sine of its original magnitude.

In other cases the vertical and horizontal angles of RE were input " "manually" to the model. In either case, once RE was specified, its components were simply mapped directly onto the corresponding components of desired change in eye position $\Delta E_{\mathrm{i}}$, to be used as initial ME

$$
\Delta E_{\mathrm{h}}=R E_{\mathrm{h}} \quad \Delta E_{\mathrm{v}}=R E_{\mathrm{v}}
$$

As described in the text, the third torsional component of $\Delta E_{\mathrm{i}}$ was set to zero to maintain Listing's law.

$$
\Delta E_{\mathrm{t}}=0
$$

The next stage of the model was simply a 3-D version of the displacement-feedback model (Jürgens et al. 1981), where instantaneous $\Delta E$ is multiplied by a gain factor within the loop to give the burst neuron $\dot{E}$ [rate of position change) command. In addition to driving the downstream motoneurons and neural integrator; each component of $\dot{E}$ was input to an integrator (that was reset to 0 after each saccade)] in the displacement-feedback loop to compute the ongoing displacement history of the saccade. Subtracting the latter from $\Delta E_{\mathrm{i}}$ gave $\Delta E$, thus closing the "local feedback loop" that accurately guided and terminated the saccade. In practice, each component of ME was exaggerated by a small fraction of its total magnitude, and then saccades were terminated when ME dropped to that fraction, so that the feedback loop would not run indefinitely.

A componentwise integration of $E$ was also performed in the oculomotor "indirect path" to compute the tonic signal. Strictly speaking, this signal encodes a torque-related vector (Schnabolk and Raphan 1994) that may deviate from the eye position vector under the circumstances of a 3-D "pulse-step mismatch." However, when velocity and position signals are correctly matched, a constant relationship between the integrator output signal and actual eye position $\underline{E}$ is maintained, so the integrator output can be correctly called $E$

$$
E_{\mathrm{t}}=\int \dot{E}_{\mathrm{t}}, \quad E_{\mathrm{v}}=\int \dot{E}_{\mathrm{v}}, \quad E_{\mathrm{h}}=\int \dot{E}_{\mathrm{h}}
$$

$E$ and $\dot{E}$ were then multiplied by internal representations of the plant elasticity $(K)$ and viscosity $(R)$ matrices, respectively, to give the correct signal-to-muscular-torque ratios. To be precise, $R$ is not the viscosity matrix but rather its equivalent in $\dot{E}$ coordinates. Like most of our predecessors (Schnabolk and Raphan 1994; Tweed and Vilis 1987), we used the same value along the diagonal elements of each matrix and zero off-diagonal elements, so this matrix multiplication amounts to scalar multiplication of $E$ and $\dot{E}$ by $k$ and $r$.

The two resulting "move" and "hold" vectors were finally summed to give the vectorial motoneuron activity $(M N)$, which was input to the linear plant model described in Tweed et al. (1994)

$$
\begin{gathered}
M N=K E+R \dot{E} \\
\underline{E}=\left(M N-\underline{k E}_{\text {old }}\right) / r \\
\underline{E}_{\text {new }}=\underline{E}_{\text {old }}+(\Delta t \times \underline{\dot{E}})
\end{gathered}
$$

where $\underline{E}$ represents the final output of the model. For purposes of plotting data it was convenient to convert this into the quaternion form to compute ongoing gaze direction relative to the head ( $\left.\underline{G}_{\text {head }}\right)$ and eye velocity $\underline{\omega}$ from $\underline{E}$ and $\underline{E}$ with the use of $E q$. 1 . In summary, this model was basically a $3-\mathrm{D}$ version of the original model of Jürgens et al. (1981), the main difference being conceptual: that burst neurons encode derivatives $(\dot{E})$ rather than angular velocity and that the plant itself implicitly provides the half-angle rule for Listing's law.

\section{Implementation of the spatial model}

Because several of the internal operations in our 3-D spatial model (Fig. $5 B$ ) were inherently rotational and multiplicative, we used quaternions to implement this model. This does not mean that this is fundamentally a "quaternion model." Rotation vectors based on tangents (e.g., Hepp et al. 1993) can also handle this rotational algebra, and, contrary to some statements (Schnabolk and Raphan 1994), so can angular position vectors, although their algebra is relatively cumbersome (Funda and Paul 1990; Tweed and Vilis 1987).

Because the spatial model required target direction rather than 
target displacement as input, $G d_{\text {eye }}$ (target direction relative to the eye) was derived from raw RE signals $(R E)$. We did this by treating $\mathrm{RE}$ as the 2-D projection of target direction on the plane orthogonal to current gaze direction, i.e., the horizontal and vertical components of $G d_{\text {eye }}(E q$. $A l)$. Again specifying that internal $G d_{\text {eye }}$ has unit length and dispensing with the optical inversion, its components could be derived from RE as follows

$$
G d_{\text {eye } 2}=-R E_{\mathrm{h}} \quad G d_{\text {eye3 }}=-R E v \quad G d_{\text {eye } 1}=\sqrt{ }\left[1-\left(R E_{\mathrm{v}}^{2}+R E_{\mathrm{h}}^{2}\right)\right]
$$

Because this step is a trivial reconstitution of $\underline{G d}$ eye, we bypassed this when computing RE from preset target directions and initial eye positions (Eq. Al). Furthermore, in cases in which RE inputs directly to the model, the more intuitive horizontal and vertical projections (Fig. 2) of $\Theta$ (referred to as, e.g., $30^{\circ}$ horizontal RE, etc.) were used, as was done in the displacement model. If one of these two angles was zero (as in the preceding simulations), then RE simply equaled the sine of the other nonzero angle. However, for cases in which both vertical and horizontal RE were nonzero, the following equations were used

$$
\begin{gathered}
G d_{\text {eye } 1}=\sqrt{ }\left[1 /\left(1+\tan ^{2} \Theta_{\mathrm{h}}+\tan ^{2} \Theta_{\mathrm{v}}\right)\right] \\
G d_{\text {eye } 2}=\tan \Theta_{\mathrm{h}}\left(G d_{\text {eye } 1}\right) \quad G d_{\text {eye } 3}=\tan \Theta_{\mathrm{v}}\left(G d_{\text {eye } 1}\right)
\end{gathered}
$$

Desired gaze relative to the head ( $\left.G d_{\text {head }}\right)$ was then computed by rotating $G d_{\text {eye }}$ by 3 -D eye position (the internal reference frame transformation) with the use of quaternion conjugation

$$
G d_{\text {head }}=E\left(G d_{\text {eye }}\right) E^{-1}
$$

A 3-D $E_{\mathrm{d}}$ was then computed by a 2-D-to-3-D transformation described by Tweed and Vilis (1990b) as the Listing's law operator, which selects the unique position on Listing's plane that satisfies that gaze direction

$$
\begin{aligned}
E_{\mathrm{d}}=\left[\sqrt{ }\left(G d_{\text {head1 }}+1\right) / 2,0,-G d_{\text {head } 3} / \sqrt{ } 2\left(G d_{\text {head1 }}+1\right),\right. \\
\left.G d_{\text {head2 }} / \sqrt{ } 2\left(G d_{\text {head1 }}+1\right)\right]
\end{aligned}
$$

Initial ME $\left(E_{\mathrm{i}}\right)$ was then computed by comparing desired eye position with an internal feedback representation of current position derived and fedback from the downstream neural integrator

$$
\Delta E_{\mathrm{i}}=E_{\mathrm{d}}-E
$$

The subsequent operations of the linear plant version of this model essentially were the same as those described in the preceding text for the displacement model. However, we also simulated a version of this model equipped with the craniotopic-torque plant, which includes true representations of $\omega$ and position-independent viscosity (Fig. 4B) (described mathematically in Tweed and Vilis 1987 ). Because this plant does not implement any special position dependencies, and requires angular velocity $(\omega)$ as the phasic input to motoneurons, the position-dependent axis tilts required by Listing's law had to be implemented neurally by converting the burst neuron signal $(\dot{E})$ into $\omega$ by reordering Eq. 1 (Fig. 5, - - -)

$$
\omega=2(\dot{E}) E^{-1}
$$

(We also simulated a displacement model equipped with a similar craniotopic plant and multiplicative position-dependent indirect path, but because this violated the simplicity of the displacement hypothesis in its purest form without adding anything interesting, these simulations have not been illustrated.)

Thus this model couches the linear operations of our displacement-feedback model between two inherently multiplicative processes (as suggested by Tweed et al. 1994): the upstream eye-tohead reference frame transformation and the downstream implementation of saccade axis tilts. Wether these tilts were implemented "neurally" or "mechanically," the final quaternion position vectors outputs were converted to angular position vectors for position plots to maintain consistency between our figures.
We thank D. Tweed, M. Smith and anonymous referees for comments that led to the final version of this paper.

This work was supported by the Human Frontiers Science Organization (D. Guitton) and the Medical Research Council (MRC) of Canada (D. Guitton and J. D. Crawford). J. D. Crawford is an Alfred P. Sloan Fellow and MRC Scholar.

Address for reprint requests: J. D. Crawford, Dept. of Psychology, York University, 4700 Keele St., North York, Ontario M3J 1P3, Canada.

Received 18 February 1997; accepted in final form 8 May 1997.

\section{REFERENCES}

Andersen, R. A., Essick, G. K., AND Siegel, R. M. Encoding of spatial location by posterior parietal neurons. Science 230: 456-458, 1985.

Clement, R. A. Natural coordinates for specification of eye movement. Vision Res. 31: 2029-2032, 1991.

CRAWFORD, J. D. The oculomotor neural integrator uses a behavior-related coordinate system. J. Neurosci. 14: 6911-6923, 1994.

Crawford, J. D. AND VIlis, T. Axes of eye rotation and Listing's law during rotations of the head. J. Neurophysiol. 65: 407-423, 1991.

CRAwFORD, J. D. AND VILIS, T. Symmetry of oculomotor burst neuron coordinates about Listing's plane. J. Neurophysiol. 68: 432-448, 1992.

Crawford, J. D. AND ViLIS, T. How does the brain deal with the problems of rotational movement? J. Mot. Behav. 27: 89-99, 1995.

Demer, J. L., Miller, J. M., Poukens, V., Vinters, H. V., And Glasgow, B. J. Evidence for fibromuscular pulleys of the recti extraocular muscles. Invest. Ophthalmol. Visual Sci. 36: 1125-1136, 1995.

Duhamel, J.-R., Colby, C. L., AND Goldberg, M. E. The updating of the representation of visual space in parietal cortex by intended eye movements. Science 255: 90-92, 1992.

Ferman, L., Collewijn, H., and Van den Berg, A. V. A direct test of Listing's law. I. Human ocular torsion measured in static tertiary positions. Vision Res. 27: 929-938, 1987.

Flanders, M., Helms Tillery, S. I., and Soechting, J. F. Early stages in a sensorimotor transformation. Behav. Brain Sci. 15: 309-362, 1992.

Freedman, E. G., Stanford, T. R., AND Sparks, D. L. Combined eye-head gaze shifts produced by electrical stimulation of the superior colliculus in rhesus monkeys. J. Neurophysiol. 76: 927-951, 1996.

Fuchs, A. F. Saccadic and smooth pursuit eye movements in the monkey. J. Physiol. (Lond.) 191: 609-631, 1967.

FUNDA, J. AND PAUL, R. P. A computational analysis of screw transformations in robotics. IEEE Trans. Robotics Automation 6: 348-356, 1990.

Goldberg, M. E. And Bruce, C. J. Primate frontal eye fields. III. Maintenance of a spatially accurate saccade signal. J. Neurophysiol. 64: 489508, 1990.

Gnadt, J. W., Bracewell, R. M., and Andersen, R. A. Sensorimotor transformations during eye movements to remembered visual targets. Vision Res. 31: 693-715, 1991.

Hallet, P. E. And Lightstone, A. D. Saccadic eye movements to flashed targets. Vision Res. 16: 107-114, 1976.

HASLWANTER, T. Mathematics of three-dimensional eye rotations. Vision Res. 35: 1727-1739, 1995.

HAusteIn, W. Considerations of Listing's law and the primary position by means of a matrix description of eye position control. Biol. Cybern. 60: 411-420, 1989.

Haustein, W. And Mittelstaedt, H. Evaluation of retinal orientation and gaze direction in the perception of the vertical. Vision Res. 30: 255-262, 1990.

von Helmholtz, H. Treatise on Physiological Optics (English translation). Translated by J.P.C. Southall. Rochester, NY: Opt. Soc. Am., 1925, vol. 3 , p. $44-51$.

HenN, V., HePp, K., AND VILIS, T. Rapid eye movement generation in the primate: physiology, pathophysiology, and clinical implications. Rev. Neurol. (Paris) 145: 540-545, 1989.

HEPP, K. Oculomotor control: Listing's law and all that. Curr. Opin. Neurobiol. 4: 862-868, 1994.

HEPP K. AND HENN V. Isofrequency curves of oculomotor neurons in the rhesus monkey. Vision Res. 25: 493-499, 1985.

Hepp, K., Van Opstal, A. J., Straumann, D., Hess, B.M.J., and Henn, V. Monkey superior colliculus represents rapid eye movements in a twodimensional motor map. J. Neurophysiol. 69: 965-979, 1993.

Hepp, K., Suzuki, J., Straumann, D., And Hess, B.J.M. On the 3-dimensional rapid eye movement generator in the monkey. In: Information 
Processing Underlying Gaze Control, edited by J. M. Delgado-Garcia, E. Godeaux, and P. P. Vidal. Oxford, UK: Pergamon, 1994, p. 65-74. Howard, I. P. Human Visual Orientation. New York: Wiley, 1982.

JÜrgens, R., BECKER, W., AND KoRnhuber, H. Natural and drug induced variations of velocity and duration of human saccadic eye movements: evidence for a control of the neural pulse generator by local feedback. Biol. Cybern. 39: 87-96, 1981.

LusChEI, E. S. AND Fuchs, A. F. Activity of brainstem neurons during eye movements of alert monkeys. J. Neurophysiol. 35: 445-461, 1972.

Miller, J. M. AND RoBins, D. Extraocular muscle sideslip and orbital geometry in monkeys. Vision Res. 27: 381-392, 1987.

Moschovakis, A. K. And Highstein, S. M. The anatomy and physiology of primate neurons that control rapid eye movements. Annu. Rev. Neurosci. 17: 465-488, 1994.

Munoz, D. P., Guitton, D., And Péllisson, D. Control of orienting gaze shifts by the tecto-reticulo-spinal system in the head free cat. III. Spatiotemporal characteristics of phasic motor discharges. J. Neurophysiol. 66: 1642-1666, 1991.

NAKAYAMA, K. Coordination of extraocular muscles. In: Basic Mechanisms of Ocular Motility and Their Clinical Implications, edited by P. Bachy-Rita and G. Lennerstrand. Oxford, UK: Pergamon, 1975, p. 193-207.

NaKAYAmA, K. Kinematics of normal and strabismic eyes. In: Vergence Eye Movements: Basic and Clinical Aspects, edited by C. M. Schor and K. J. Ciuffreda. Boston, MA: Butterworths, 1983, p. 543-564.

Robinson, D. A. Eye movements evoked by collicular stimulation in the alert monkey. Vision Res. 12: 1795-1808, 1972.

Robinson, D. A. Oculomotor control signals. In: Basic Mechanisms of Ocular Motility and Their Clinical Implications, edited by P. Bach-yRita and G. Lennerstrand. Oxford, UK: Pergamon, 1975, p. 337-374.

Robinson, D. A. Use of matrices in analyzing the three-dimensional behaviour of the vestibulo-ocular reflex. Biol. Cybern. 46: 53-66, 1982.

Robinson, D. A. AND ZEE, D. S. Theoretical considerations of the function and circuitry of various rapid eye movements. In: Progress in Oculomotor Research, Developments in Neuroscience, edited by A. F. Fuchs and W. Becker. New York: Elsevier/North-Holland, 1981, vol. 12, p. 3-9.

SCHILLER, P. H. AND STRYKER, M. Single unit recording and stimulation in superior colliculus of alert rhesus monkey. J. Neurophysiol. 35: 915924, 1972.

Schlag, J., Schlag-Rey, M., and Dassonville, P. Interactions between natural and electrically evoked saccades. II. At what time is eye position sampled as a reference for the localization of a target? Exp. Brain Res. 76: 548-558, 1989.

SCHNABOLK, C. AND RaPhan, T. Modelling three-dimensional velocity to position transformation in oculomotor control. J. Neurophysiol. 71: 623638, 1994.

SCUDDER, C. A. A new local feedback model of the saccadic burst generator. J. Neurophysiol. 59: 1455-1475, 1988.

SPARKS, D. L. Neural cartography: sensory and motor maps in the superior colliculus. Brain Behav. Evol. 31: 49-56, 1988.

SparKs, D. L. AND MAYs, L. E. Spatial localization of saccade targets. I. Compensation for stimulation-induced perturbations in eye position. $J$. Neurophysiol. 49: 45-63, 1983.

Sparks, D. L., Mays, L. E., AND Porter, J. D. Eye movements induced by pontine stimulation: interaction with visually triggered saccades. $J$. Neurophysiol. 58: 300-318, 1987.
StANFord, T. R. AND Sparks, D. L. Systematic errors for saccades to remembered targets: evidence for a dissociation between saccade metrics and activity in the superior colliculus. Vision Res. 34: 93-106, 1994.

Straumann, D., Zee, D. S., Solomon, D., Lasker, A. G., And Roberts, D. C. Transient torsion and cyclovergence during and after saccades. Vision Res. 35: 3321-3335, 1995.

Suzuki, Y., Buttner-Ennever, J. A., Straumann, D., Hepp, K., Hess, B.J.M., AND HENN, V. Deficits in torsional and vertical rapid eye movements and shift of Listing's plane after uni- and bilateral lesions of the rostral interstitial nucleus of the medial longitudinal fasciculus. Exp. Brain Res. 106: 215-232, 1995.

Tweed, D., Glenn, B., AND Vilis, T. Eye head coordination during large gaze shifts. J. Neurophysiol. 73: 766-779, 1995.

Tweed, D., Misslisch, H., AND FetTer, M. Testing models of the oculomotor velocity to position transformation. J. Neurophysiol. 72: 1425-1429, 1994.

TwEED, D. AND VILIS, T. Implications of rotational kinematics for the oculomotor system in three dimensions. J. Neurophysiol. 58: 832-849, 1987.

TweED, D. AND ViLIs, T. Geometric relations of eye position and velocity vectors during saccades. Vision Res. 30: 111-127, 1990a.

TweEd, D. AND VILIS, T. The superior colliculus and spatiotemporal translation in the saccadic system. Neural Networks 3: 75-86, 1990b.

VAn Opstal, A. J. AND HePP, K. A novel interpretation for the collicular role in saccade generation. Biol. Cybern. 73: 431-445, 1995.

Van Opstal, A. J., Hepp, K., Hess, B.J.M., Straumann, D., and Henn, V. Two- rather than three-dimensional representation of saccades in monkey superior colliculus. Science 252: 1313-1315, 1991.

Van Opstal, A. J., Hepp, K., Suzuki, Y., and Henn, V. Influence of eye position on activity in monkey superior colliculus. J. Neurophysiol. 74: 1593-1610, 1995.

Van Opstal, A. J., Hepp, K., Suzuki, Y., and Henn, V. Role of monkey nucleus reticularis tegmenti pontis in the stabilization of Listing's plane. J. Neurosci. 16: 7284-7296, 1996.

Waitzman, D. M., Ma, T. P., Optican, L. M., And Wurtz, R. H. Superior colliculus neurons mediate the dynamic characteristics of saccades. $J$. Neurophysiol. 66: 1716-1737, 1991.

WestheIMER, G. Kinematics of the eye. J. Opt. Soc. Am. 47: 967-974, 1957.

WeSTHEIMER, G. Saccadic eye movements. In: The Oculomotor System and Brain Functions, edited by V. Zikmund. Bratislava, Czech Republic: Slovak Acad. Sciences, 1973, p. 57-77.

White, J. M., SPARKs, D. L., AND StANFord, T. R. Saccades to remembered target locations: an analysis of systematic and variable errors. Vision Res. 34: 79-92, 1994.

Woodwoth, R. S. The accuracy of voluntary movement. Psychol. Rev. Monogr. Suppl. 3: 1899.

Wurtz, R. H. and Albano, J. E. Visual-motor function of the primate superior colliculus. Annu. Rev. Neurosci. 3: 189-226, 1980.

Yarbus, A. L. Eye Movements and Vision. New York: Plenum, 1967.

Zee, D. S., Optican, L. M., Cook, J. D., Robinson, D. A., and Engel, W. K. Slow saccades in spinocerebellar degeneration. Arch. Neurol. 33: 243-251, 1976.

ZIPSER, D. AND ANDERSEN, R. A. A back-propogation programmed network that simulates response properties of a subset of posterior parietal neurons. Nature 331: 679-684, 1988. 